\title{
Lipoprotein Subfractions and Glucose Homeostasis in Prediabetes and Diabetes in Taiwan
}

\author{
Hung Hsu ${ }^{1}$, Powen Hsu², Ming-Hui Cheng ${ }^{3}$, Yasuki Ito ${ }^{4}$, Eiichiro Kanda ${ }^{5}$, Ernst J Schaefer ${ }^{6}$ and Masumi $\mathrm{Ai}^{1}$ \\ ${ }^{1}$ Department of Insured Medical Care Management, Graduate School of Medical and Dental Sciences, Tokyo Medical and Dental \\ University (TMDU), Tokyo, Japan \\ ${ }^{2}$ Department of General Internal Medicine, Lo-Hsu Medical Foundation Lotung Poh-Ai Hospital, Lotung, Yilan, Taiwan \\ ${ }^{3}$ Department of Laboratory Medicine, Lo-Hsu Medical Foundation Lotung Poh-Ai Hospital, Lotung, Yilan, Taiwan \\ ${ }^{4}$ Research and Development Center, Denka-Seiken Company, Ltd., Tokyo, Japan \\ ${ }^{5}$ Department of Medical Science, Kawasaki Medical School, Kurashiki, Japan \\ ${ }^{6}$ Cardiovascular Nutrition Laboratory, Human Nutrition Research Center on Aging at Tufts University, and Tufts University School \\ of Medicine, Boston, MA, USA
}

Aims: Prediabetes and diabetes are associated with increased insulin resistance and decreased insulin production, dyslipidemia, and increased cardiovascular disease (CVD) risk. Our goals were to assess lipoprotein subfractions using novel assays in such subjects.

Methods: Fasting normal, prediabetic, and diabetic Taiwanese men and women $(n=2,049)$ had their serum glucose, glycosylated hemoglobin, insulin, total cholesterol (TC), triglycerides (TG), high-density lipoprotein cholesterol (HDL-C), HDL3-C, apolipoprotein E-HDL-C, direct low-density lipoprotein cholesterol (LDL-C), small dense LDL-C (sdLDL-C), LDL-TG, and remnant lipoprotein cholesterol (RLP-C) levels measured using novel assays. HDL2-C, LDL-C, and large-buoyant LDL-C (lbLDL-C) were calculated.

Results: Prediabetic male and female subjects had significantly higher levels of TG, RLP-C, sdLDL-C, the sdLDL-C/LDL-C ratio, and LDL-TG than normal subjects, and statin treatment abolished this effect in men, but not in women. Diabetic male and female subjects had significantly higher TG and sdLDL-C/LDL-C ratios, and significantly lower levels of HDL-C, HDL2-C, HDL3-C, and apoE HDL-C than normal subjects, as did prediabetic women. Median direct LDL-C levels were $>100 \mathrm{mg} / \mathrm{dL}$ in all groups, even in those receiving statin therapy. Calculated LDL-C significantly underestimated direct LDL-C by $>10 \%$ in diabetic subjects.

Conclusions: Our data indicate that prediabetic subjects were more likely to have significantly elevated RLP-C, sdLDL-C, and LDL-TG, while diabetic subjects were more likely to have significantly decreased HDL-C, HDL2C, HDL3-C, and apoE HDL-C than normal subjects, and calculated LDL-C significantly underestimated their direct LDL-C. In our view, direct LDL-C and sdLDL-C should be measured and optimized in both diabetic and prediabetic subjects to reduce CVD risk.

Key words: Low-density lipoprotein triglyceride, Small dense low-density lipoprotein cholesterol, Type 2 diabetes mellitus, Prediabetes, Lipid-lowering medication

\section{Introduction}

Diabetes mellitus is a major risk factor for cardiovascular disease (CVD), and is associated with dyslipidemia characterized by increased serum levels of tri- glycerides (TG), remnant lipoprotein cholesterol (RLP$\mathrm{C})$, and small dense low-density lipoproteins (sdLDL), and decreased levels of high-density lipoprotein cholesterol (HDL-C) ${ }^{1-4)}$. Subjects with prediabetes are also at increased CVD risk, and often have dyslipidemia ${ }^{5)}$.

Address for correspondence: Masumi Ai, Department of Insured Medical Care Management, Graduate School of Medical and Dental Sciences, Tokyo Medical and Dental University (TMDU), 1-5-45 Yushima, Bunkyo-ku, Tokyo, 113-8519, Japan E-mail: ai.vasc@tmd.ac.jp

Received: December 2, 2018 Accepted for publication: January 14, 2019

Copyright@2019 Japan Atherosclerosis Society

This article is distributed under the terms of the latest version of CC BY-NC-SA defined by the Creative Commons Attribution License. 
Significant CVD risk factors in patients with diabetes include hypertension, smoking, increased levels of serum glucose and LDL-C, and decreased levels of HDL- $\mathrm{C}^{6}$. Lowering LDL-C levels with statin therapy has been associated with significant reductions in CVD events in patients with diabetes ${ }^{7-11)}$.

In the United States, it has been recommended that all patients with established CVD and diabetes over the age of 40 years with LDL-C levels $>70 \mathrm{mg} /$ $\mathrm{dL}$, an LDL-C value $\geq 190 \mathrm{mg} / \mathrm{dL}$, or a ten-year CVD risk $\geq 7.5 \%$ be placed on statin therapy in addition to lifestyle modification in order to achieve a significant LDL-C reduction ${ }^{12-14)}$. More recently, it has been suggested that in addition to statins, the use of ezetimibe, and/or proprotein convertase subtilisin kexin 9 inhibitors can be considered to reduce LDL-C levels to $<70$ $\mathrm{mg} / \mathrm{dL}$ in CVD patients and $<100 \mathrm{mg} / \mathrm{dL}$ in highrisk patients including patients with diabetes ${ }^{15}, 16$. In the United States, there are no recommendations with regard to TG targets ${ }^{12-16)}$. In Taiwan, current recommendations with regard to LDL-C targets of therapy are the same as in the United States, but it was also recommended that TG levels be lowered to $<150 \mathrm{mg} /$ $\mathrm{dL}^{17}{ }^{18)}$. In Japan, it was recommended that CVD patients have their LDL-C values lowered to $<100$ $\mathrm{mg} / \mathrm{dL}$, and $<120 \mathrm{mg} / \mathrm{dL}$ for diabetics, and for both groups to get TG levels lowered to $<150 \mathrm{mg} / \mathrm{dL}^{19)}$. In the Action to Control Cardiovascular Risk in Diabetes (ACCORD) Trial, it was documented that diabetic subjects on statin therapy did not get additional benefit from the addition of fenofibrate therapy, unless their TG levels were $>204 \mathrm{mg} / \mathrm{dL}$ and their HDL-C levels were $<35 \mathrm{mg} / \mathrm{dL}$. This latter group got a $28 \%$ risk reduction when fenofibrate was added to statin therapy as compared to placebo therapy ${ }^{20)}$.

It has long been known that hypertriglyceridemia is associated with decreased HDL-C values, as well as increased levels of sdLDL-C ${ }^{21-23)}$. This latter parameter can now be readily measured with an automated assay ${ }^{24)}$. In addition, it has been reported that elevated levels of RLP-C, sdLDL-C, and LDL-TG, and decreased levels of HDL-C and its subfractions have been associated with increased CVD risk ${ }^{21-33)}$. However, these new assays have not been applied to a large diabetic and prediabetic population, especially in an Asian population.

\section{Aim}

Our purpose in the present study was to evaluate potential differences in the levels of RLP-C, direct LDL-C, large-buoyant LDL-C (lbLDL-C), sdLDL-C, HDL-C, HDL3-C, HDL2-C, and apoE-HDL-C among individuals with type 2 diabetes, prediabetes, or normal glucose tolerance using novel automated assays.
Another goal of this investigation was to examine relationships between lipoprotein subfractions and measures of glucose homeostasis, including insulin, and measures of insulin resistance and production in normal, prediabetic, and diabetic subjects in a large Asian population.

\section{Methods}

Our study population consisted of 2,049 men and women living in Yi-lan County in northern Taiwan. Residents of this province are native to the island of Taiwan and are not recent immigrants from mainland China or other parts of Asia. All subjects agreed to participate in this study using a standard study protocol and informed consent approved by the research committees of Luodong Poh-ai Hospital, Luodong, Taiwan and the School of Medicine, Tokyo Medical and Dental University, Tokyo, Japan. (M2000-2350).

We recruited normal, prediabetic, and diabetic subjects from participants after testing and participation in a standard health examination as previously described $^{34)}$. Subjects were classified as normal if their fasting serum glucose value was $<100 \mathrm{mg} / \mathrm{dL}$, as prediabetic if their value was in the $100-125 \mathrm{mg} / \mathrm{dL}$ range, and as diabetic if their value was $>125 \mathrm{mg} / \mathrm{dL}$ using the standard criteria. In order to increase our diabetic subjects, subjects with diabetes were also recruited from a clinic at Luodong Poh-ai Hospital, and were required to have a history of diabetes without any change in treatment including medications for at least 6 months. All diabetic patients received standardized therapies consistent with national Taiwanese, American Diabetes Association and European Association for the Study of Diabetes guidelines. All participants were classified as being obese if their body mass index (BMI) was $\geq 27 \mathrm{~kg} / \mathrm{m}^{2}$ (Taiwanese criteria), or in some analyses (see Supplemental Tables) as $\geq 25 \mathrm{~kg} / \mathrm{m}^{2}$ (Japanese criteria) ${ }^{35-37)}$.

Subjects being treated for thyroid disease, or those with laboratory evidence of liver disease (transaminase value $>$ three times the upper limits of normal), or kidney disease (creatinine $>2.0 \mathrm{mg} / \mathrm{dL}$ ), or those receiving insulin therapy were excluded from the study. Among the participants, 667 were in the normal group, 345 were in the prediabetic group, and 1,037 were in the diabetic group. In all subjects, a standard history about health status and medication use was obtained, and a physical examination was carried out including measurements of height, weight, and blood pressure. Subjects with a significant history of CVD were excluded from this analysis.

Blood samples were collected from all participants after an overnight fast of 12 hours or more. Serum 
Table 1. Characteristics of all male subjects $(n=1030)^{*}$

\begin{tabular}{|c|c|c|c|c|c|c|}
\hline Variable & $\begin{array}{c}\text { NGT } \\
n=277\end{array}$ & $\begin{array}{l}\text { PreDM } \\
n=200\end{array}$ & $\begin{array}{c}\text { DM } \\
n=553\end{array}$ & \multicolumn{3}{|c|}{$P$ value } \\
\hline Age, year & $55.8(20.3)$ & $60.6(18.6)$ & $61.5(14.6)$ & 0.002 & 0.098 & 0.000 \\
\hline BMI, $\mathrm{kg} / \mathrm{m}^{2}$ & $24.2(4.2)$ & $25.3(3.9)$ & $26.1(4.8)$ & 0.001 & 0.001 & 0.000 \\
\hline Obesity Prevalence & $50(18.1 \%)^{* *}$ & $53(26.5 \%)^{* *}$ & $217(39.2 \%)^{* *}$ & 0.001 & 0.001 & 0.000 \\
\hline Fasting Glucose, mg/dL & $93.0(8.0)$ & $105.0(9.0)$ & $133.0(40.0)$ & 0.000 & 0.000 & 0.000 \\
\hline HbAlc, \% & $5.5(0.4)$ & $5.8(0.4)$ & $6.9(1.3)$ & 0.000 & 0.000 & 0.000 \\
\hline Insulin, & $8.0(1.5)$ & $10.6(21.2)$ & $10.7(10.4)$ & 0.000 & 0.823 & 0.000 \\
\hline HOMA-IR & $1.8(1.7)$ & $2.8(2.2)$ & $3.5(3.6)$ & 0.000 & 0.000 & 0.000 \\
\hline НOMА- $\beta$ & $100.4(90.4)$ & $86.5(58.7)$ & $58.9(65.8)$ & 0.018 & 0.000 & 0.000 \\
\hline RLP-C, mg/dL & $6.3(7.3)$ & $8.7(10.7)$ & $6.3(9.1)$ & 0.001 & 0.001 & 0.984 \\
\hline LDL-C, mg/dL & $113.3(46.7)$ & $111.8(43.7)$ & $106.4(32.7)$ & 0.880 & 0.032 & 0.008 \\
\hline calculated LDL-C, mg/dL & $115.2(51.1)$ & $109.4(43.8)$ & $93.2(35.7)$ & 0.176 & 0.000 & 0.000 \\
\hline sdLDL-C, mg/dL & $29.5(22.2)$ & $34.5(25.0)$ & $32.7(20.4)$ & 0.018 & 0.251 & 0.052 \\
\hline sdLDL-C/LDL-C ratio & $0.260(0.111)$ & $0.305(0.150)$ & $0.299(0.153)$ & 0.000 & 0.896 & 0.000 \\
\hline lbLDL-C, mg/dL & $80.1(34.1)$ & $78.0(33.8)$ & $73.2(27.0)$ & 0.094 & 0.028 & 0.000 \\
\hline LDL-TG, mg/dL & $22.2(11.0)$ & $23.3(10.1)$ & $19.4(7.7)$ & 0.006 & 0.000 & 0.000 \\
\hline Non-HDL-C, mg/dL & $140.7(54.8)$ & $138.7(41.6)$ & $122.7(38.1)$ & 0.945 & 0.000 & 0.000 \\
\hline HDL-C, mg/dL & $51.3(17.2)$ & $50.3(16.1)$ & $46.0(13.5)$ & 0.460 & 0.000 & 0.000 \\
\hline $\mathrm{HDL}_{2}-\mathrm{C}, \mathrm{mg} / \mathrm{dL}$ & $26.9(13.3)$ & $27.0(10.0)$ & $25.1(8.1)$ & 0.447 & 0.069 & 0.005 \\
\hline
\end{tabular}

* Data are expressed as median (interquartile range).

** Data are expressed as number of obese participant (percentage of obese participants) $\left(\mathrm{BMI} \geq 27 \mathrm{~kg} / \mathrm{m}^{2}\right)$.

Apo, apolipoprotein; BMI, body mass index; BP, blood pressure; DM, diabetes mellitus; HbA1c, glycated hemoglobin; HOMA-IR, homeostatic model assessment on Insulin Resistance; HOMA- $\beta$, homeostatic model assessment on beta-cell; HDL-C, HDL cholesterol; lbLDL-C, large buoyant LDL cholesterol; LDL-C, LDL cholesterol; NGT, normal glucose tolerance; preDM, pre-diabetes mellitus; RLP-C, remnant lipoprotein cholesterol; sdLDL-C, small dense LDL cholesterol; TG, triglycerides

glucose was measured using the hexokinase method, glycosylated hemoglobin using a turbidimetric inhibition immunoassay as previously described ${ }^{34)}$. Insulin levels were measured by chemiluminescence assay on a Siemens Centaur automated platform (Siemens Healthineers, Germany) with intra- and inter-assay coefficients of variation $(\mathrm{CVs})$ of $<5 \%$. Serum creatinine, liver transaminases, TC, TG, HDL-C, HDL3-C, apoEHDL-C, direct LDL-C, sdLDL-C, LDL-TG, and RLP-C were measured by automated standardized enzymatic analysis on a Cobas C501 analyzer (Roche Diagnostics, Germany). The specialized lipid assay kits were provided by the Denka-Seiken Corporation (Tokyo, Japan) as previously described ${ }^{24,31,32,38-43)}$. All specialized lipid assays had within and between run CVs were $<6.0 \%$, and in most cases $<3.0 \%$. Homeostasis model assessment of insulin resistance (HOMA-IR) and homeostasis model assessment of insulin production (HOMA- $\beta$ ) were calculated from glucose and insulin levels using the following formulas: $\mathrm{HOMA}_{\beta}=$ [(insulin in $\mu \mathrm{U} / \mathrm{mL} \times 360) /($ glucose in $\mathrm{mg} / \mathrm{dL}-63)] \%$ ), and $\mathrm{HOMAIR}_{\mathrm{IR}}=$ [glucose in $\mathrm{mg} / \mathrm{dL} \times($ insulin in $\mu \mathrm{U} /$ $\mathrm{mL} / 405)]^{44,45)}$. lbLDL-C were calculated by subtracting sdLDL-C from direct LDL-C. HDL2-C were calculated by subtracting HDL3-C from HDL-C. LDL-C was calculated using the Friedewald formula by subtracting the sum of HDL-C and TG/5 from TC.

Data are presented as the median values with inter-quartile ranges since many variables were not normally distributed. All statistical analyses were performed using SPSS version 22 (IBM, Armonk, NY). Mann-Whitney $U$ testing was used to assess the sta- 
Table 2. Characteristics of all female subjects $(n=1019)^{*}$

\begin{tabular}{|c|c|c|c|c|c|c|}
\hline \multirow[b]{2}{*}{ Variable } & \multirow{2}{*}{$\begin{array}{c}\text { NGT } \\
n=390\end{array}$} & \multirow{2}{*}{$\begin{array}{l}\text { PreDM } \\
n=145\end{array}$} & \multirow{2}{*}{$\begin{array}{c}\mathrm{DM} \\
n=484\end{array}$} & \multicolumn{3}{|c|}{$P$ value } \\
\hline & & & & $\begin{array}{l}\text { NGT vs } \\
\text { PreDM }\end{array}$ & $\begin{array}{l}\text { PreDM } \\
\text { vs DM }\end{array}$ & $\begin{array}{c}\text { DM vs } \\
\text { NGT }\end{array}$ \\
\hline Age, year & $51.7(16.4)$ & $61.5(18.8)$ & $63.7(12.3)$ & 0.000 & 0.018 & 0.000 \\
\hline BMI, $\mathrm{kg} / \mathrm{m}^{2}$ & $22.8(4.0)$ & $23.8(4.6)$ & $25.5(5.0)$ & 0.001 & 0.000 & 0.000 \\
\hline Obesity Prevalence & $36(9.2 \%)^{* *}$ & $31(21.4 \%)^{* *}$ & $172(35.5 \%)^{* *}$ & 0.001 & 0.000 & 0.000 \\
\hline Systolic BP, mmHg & $118.0(19.0)$ & $123.0(16.0)$ & $130.0(14.0)$ & 0.003 & 0.000 & 0.000 \\
\hline Diastolic BP, mmHg & $69.0(14.0)$ & $71.0(14.3)$ & $70.0(10.0)$ & 0.028 & 0.076 & 0.000 \\
\hline Fasting Glucose, mg/dL & $90.0(9.0)$ & $105.0(11.0)$ & $132.0(41.0)$ & 0.000 & 0.000 & 0.000 \\
\hline HbAlc, \% & $5.5(0.4)$ & $5.9(0.5)$ & $7.0(1.4)$ & 0.000 & 0.000 & 0.000 \\
\hline Insulin, & $8.3(5.8)$ & $12.7(8.3)$ & $13.2(10.1)$ & 0.000 & 0.251 & 0.000 \\
\hline HOMA-IR & $1.8(1.3)$ & $3.3(2.4)$ & $4.3(4.0)$ & 0.000 & 0.000 & 0.000 \\
\hline HOMA- $\beta$ & $113.1(76.4)$ & $97.7(67.8)$ & $66.1(68.0)$ & 0.004 & 0.000 & 0.000 \\
\hline \multicolumn{7}{|l|}{ Lipids and Lipoproteins } \\
\hline Total cholesterol, mg/dL & $197.0(48.8)$ & $194.0(42.0)$ & $175.0(36.3)$ & 0.254 & 0.000 & 0.000 \\
\hline Triglycerides, mg/dL & $86.5(58.8)$ & $116.0(94.0)$ & $119.0(82.3)$ & 0.000 & 0.983 & 0.000 \\
\hline $\mathrm{RLP}-\mathrm{C}, \mathrm{mg} / \mathrm{dL}$ & $5.3(4.7)$ & $7.9(9.8)$ & $6.2(7.6)$ & 0.000 & 0.000 & 0.000 \\
\hline LDL-C, mg/dL & $117.0(39.6)$ & $114.3(42.7)$ & $106.6(35.5)$ & 0.448 & 0.007 & 0.000 \\
\hline calculated LDL-C, mg/dL & $116.7(42.9)$ & $112.3(42.1)$ & $95.1(34.7)$ & 0.098 & 0.000 & 0.000 \\
\hline sdLDL-C, mg/dL & $27.3(17.1)$ & $33.5(21.4)$ & $31.5(19.6)$ & 0.000 & 0.165 & 0.000 \\
\hline sdLDL-C/LDL-C ratio & $0.231(0.085)$ & $0.287(0.127)$ & $0.290(0.129)$ & 0.000 & 0.813 & 0.000 \\
\hline $\mathrm{lbLDL}-\mathrm{C}, \mathrm{mg} / \mathrm{dL}$ & $87.9(28.0)$ & $80.6(29.3)$ & $74.5(26.5)$ & 0.001 & 0.002 & 0.000 \\
\hline LDL-TG, mg/dL & $21.2(10.0)$ & $25.2(10.5)$ & $20.9(8.3)$ & 0.000 & 0.000 & 0.857 \\
\hline Non-HDL-C, mg/dL & $134.4(48.1)$ & $139.3(41.7)$ & $121.6(36.3)$ & 0.335 & 0.000 & 0.000 \\
\hline HDL-C, mg/dL & $59.2(18.4)$ & $52.2(17.9)$ & $52.5(15.8)$ & 0.000 & 0.962 & 0.000 \\
\hline $\mathrm{HDL}_{2}-\mathrm{C}, \mathrm{mg} / \mathrm{dL}$ & $34.0(14.7)$ & $28.9(11.8)$ & $29.4(11.0)$ & 0.000 & 0.171 & 0.000 \\
\hline $\mathrm{HDL}_{3}-\mathrm{C}, \mathrm{mg} / \mathrm{dL}$ & $25.0(4.7)$ & $23.9(5.4)$ & $22.8(5.6)$ & 0.018 & 0.001 & 0.000 \\
\hline ApoE-HDL, mg/dL & $5.7(2.3)$ & $5.0(2.0)$ & 4.7 (1.9) & 0.000 & 0.020 & 0.000 \\
\hline
\end{tabular}

* Data are expressed as median (interquartile range).

** Data are expressed as number of obese participant (percentage of obese participants) $\left(\mathrm{BMI} \geq 27 \mathrm{~kg} / \mathrm{m}^{2}\right)$.

Apo, apolipoprotein; BMI, body mass index; BP, blood pressure; DM, diabetes mellitus; HbA1c, glycated hemoglobin; HOMA-IR, homeostatic model assessment on Insulin Resistance; HOMA- $\beta$, homeostatic model assessment on beta-cell; HDL-C, HDL cholesterol; lbLDL-C, large buoyant LDL cholesterol; LDL-C, LDL cholesterol; NGT, normal glucose tolerance; preDM, pre-diabetes mellitus; RLP-C, remnant lipoprotein cholesterol; sdLDL-C, small dense LDL cholesterol; TG, triglycerides; non HDL-C, non HDL cholesterol;

tistical significance of differences for assessed variables between groups. Pearson's correlation coefficients were used for assessing the significance of correlations between variables. A $P$ value of $<0.05$ was considered statistically significant.

\section{Results}

Data on all male $(n=1,030)$ and female $(n=1,019)$ participants are shown in Tables 1 and 2, respectively. Since this study focused on lipoprotein subfractions, data on male $(n=605)$ and female $(n=622)$ subjects off lipid-lowering medications are shown in Table 3 and 4, respectively, while data on men $(n=347)$ and women $(n=339)$ on statin therapy are shown in Tables 5 and 6 , respectively. In addition, we tabulated relative percentage differences between normal, prediabetic, and diabetic male and female subjects for all lipoprotein subfractions in all subjects, those off statins, and in those on statin therapy in Figs.1, 2, and 3, respectively. In addition, we have analyzed the effects of obesity classified as $\geq 27 \mathrm{~kg} / \mathrm{m}^{2}$ using the Taiwanese criteria in normal prediabetic and diabetic subgroups for men in Table 7, and for women in Table 8. Comparisons of normal, prediabetic, and diabetic men and women with and without obesity classified as $\geq 25 \mathrm{~kg} /$ $\mathrm{m}^{2}$ using Japanese criteria are shown in Supplemental Tables 1 and 2. Comparison of variables between participants with and without statin use are shown in Supplemental Table 3-5 (normal group in Supplemental Table 3, prediabetic group in Supplemental Table 4, and DM group in Supplemental Table 5). 
Table 3. Characteristics of male subjects not on lipid medications $(n=605)^{*}$

\begin{tabular}{|c|c|c|c|c|c|c|}
\hline Variable & $\begin{array}{c}\text { NGT } \\
n=207\end{array}$ & $\begin{array}{l}\text { PreDM } \\
n=117\end{array}$ & $\begin{array}{c}\text { DM } \\
n=281\end{array}$ & \multicolumn{3}{|c|}{$P$ value } \\
\hline Age, year & $52.2(18.3)$ & $54.5(18.5)$ & $61.9(13.4)$ & 0.017 & 0.000 & 0.000 \\
\hline BMI, $\mathrm{kg} / \mathrm{m}^{2}$ & $23.9(4.1)$ & $25.3(4.0)$ & $25.4(4.7)$ & 0.000 & 0.710 & 0.000 \\
\hline Obesity Prevalence & $36(17.4 \%)^{* *}$ & $36(30.8 \%)^{* *}$ & $94(33.5 \%)^{* *}$ & 0.000 & 0.710 & 0.000 \\
\hline Fasting Glucose, mg/dL & $92.0(7.5)$ & $105.0(10.0)$ & $134.0(35.0)$ & 0.000 & 0.000 & 0.000 \\
\hline HbAlc, \% & $5.5(0.4)$ & $5.8(0.5)$ & $6.9(1.1)$ & 0.000 & 0.000 & 0.000 \\
\hline Insulin, & $7.9(7.4)$ & $10.6(8.9)$ & $10.5(10.7)$ & 0.000 & 0.934 & 0.000 \\
\hline HOMA-IR & $1.8(1.6)$ & $2.7(2.3)$ & $3.3(3.7)$ & 0.000 & 0.006 & 0.000 \\
\hline НOMА- $\beta$ & $99.5(96.2)$ & $85.1(63.5)$ & $55.2(61.7)$ & 0.008 & 0.000 & 0.000 \\
\hline RLP-C, mg/dL & $6.3(8.2)$ & $10.1(11.6)$ & $5.6(8.0)$ & 0.001 & 0.000 & 0.061 \\
\hline LDL-C, mg/dL & $116.7(44.8)$ & $115.5(40.9)$ & $107.2(30.1)$ & 0.686 & 0.003 & 0.000 \\
\hline calculated LDL-C, mg/dL & $117.7(47.3)$ & $113.5(39.9)$ & $94.1(31.0)$ & 0.103 & 0.000 & 0.000 \\
\hline sdLDL-C, mg/dL & $30.7(22.9)$ & $34.9(26.1)$ & $31.8(18.8)$ & 0.075 & 0.032 & 0.957 \\
\hline sdLDL-C/LDL-C ratio & $0.256(0.118)$ & $0.310(0.164)$ & $0.288(0.139)$ & 0.004 & 0.386 & 0.003 \\
\hline lbLDL-C, mg/dL & $84.9(33.0)$ & $79.7(32.5)$ & $74.6(25.2)$ & 0.115 & 0.021 & 0.000 \\
\hline LDL-TG, mg/dL & $22.1(11.3)$ & $23.4(10.9)$ & $18.1(6.6)$ & 0.026 & 0.000 & 0.000 \\
\hline Non HDL-C, mg/dL & $142.9(52.2)$ & $141.6(33.2)$ & $122.8(30.9)$ & 0.815 & 0.000 & 0.000 \\
\hline HDL-C, mg/dL & $51.6(18.1)$ & $51.4(18.0)$ & $46.0(13.6)$ & 0.551 & 0.003 & 0.000 \\
\hline $\mathrm{HDL}_{2}-\mathrm{C}, \mathrm{mg} / \mathrm{dL}$ & $27.6(13.0)$ & $26.3(12.4)$ & $25.2(8.3)$ & 0.448 & 0.238 & 0.015 \\
\hline
\end{tabular}

* Data are expressed as median (interquartile range).

** Data are expressed as number of obese participant (percentage of obese participants) $\left(\mathrm{BMI} \geq 27 \mathrm{~kg} / \mathrm{m}^{2}\right)$.

Apo, apolipoprotein; BMI, body mass index; BP, blood pressure; DM, diabetes mellitus; HbA1c, glycated hemoglobin; HOMA-IR, homeostatic model assessment on Insulin Resistance; HOMA- $\beta$, homeostatic model assessment on beta-cell; HDL-C, HDL cholesterol; lbLDL-C, large buoyant LDL cholesterol; LDL-C, LDL cholesterol; NGT, normal glucose tolerance; preDM, pre-diabetes mellitus; RLP-C, remnant lipoprotein cholesterol; sdLDL-C, small dense LDL cholesterol; TG, triglycerides

Correlations between variables for all subjects, men and women are shown in Supplemental Tables 6, 7, and 8.

For all subjects and for those not receiving or receiving statin therapy, the median BMI, obesity prevalence, systolic blood pressure, serum glucose, HbA1c, serum insulin, and calculated insulin resistance (HOMAIR) were all significantly higher in prediabetic and diabetic subjects than in normal subjects. In contrast, median calculated insulin production $\left(\mathrm{HOMA}_{\beta}\right)$ values were significantly lower in prediabetic and diabetic subjects than in normal, especially in those with diabetes (see Tables 1-6). Interestingly serum insulin levels were not significantly different between prediabetic and diabetic subjects in all categories, but there were significantly higher HOMAIr values and signifi- cantly lower $\mathrm{HOMA}_{\beta}$ values in diabetic subjects than in normal subjects.

In all male prediabetic subjects, TG, RLP-C, sdLDL-C, sdLDL-C/LDL-C ratio, and LDL-TG values were all significantly higher than in normal subjects (see Table 1). In male prediabetics not on statin therapy, TG, sdLDL-C/LDL-C ratio, and LDL-TG values were all significantly higher than in normal subjects (see Table 3). In male prediabetics on statin therapy, all these significant differences were abolished (see Table 5). In all female prediabetic subjects, TG, RLPC, sdLDL-C, sdLDL-C/LDL-C ratio, and LDL-TG values were all significantly higher than in normal subjects, while lbLDL-C, HDL-C, HDL2-C, and apoEHDL-C were all significantly lower than in normals (see Table 2). In female prediabetic subjects off statin 
Table 4. Characteristics of female subjects not on lipid medications $(n=622) *$

\begin{tabular}{|c|c|c|c|c|c|c|}
\hline Variable & $\begin{array}{c}\text { NGT } \\
n=324\end{array}$ & $\begin{array}{c}\text { PreDM } \\
n=90\end{array}$ & $\begin{array}{c}\text { DM } \\
n=208\end{array}$ & \multicolumn{3}{|c|}{$P$ value } \\
\hline Age, year & $49.3(12.9)$ & $54.7(19.2)$ & $63.6(11.9)$ & 0.000 & 0.000 & 0.000 \\
\hline BMI, $\mathrm{kg} / \mathrm{m}^{2}$ & $22.5(4.0)$ & $24.1(5.2)$ & $24.8(4.8)$ & 0.000 & 0.285 & 0.000 \\
\hline Obesity Prevalence & $25(7.7 \%)^{* *}$ & $23(25.6 \%)^{* *}$ & $57(27.4 \%)^{* *}$ & 0.000 & 0.285 & 0.000 \\
\hline Fasting Glucose, mg/dL & $89.5(8.0)$ & $105.0(11.0)$ & $132.0(39.5)$ & 0.000 & 0.000 & 0.000 \\
\hline HbAlc, \% & $5.4(0.4)$ & $5.8(0.7)$ & $7.0(1.2)$ & 0.000 & 0.000 & 0.000 \\
\hline Insulin, & $7.9(5.6)$ & $11.9(8.2)$ & $12.0(9.4)$ & 0.000 & 0.669 & 0.000 \\
\hline HOMA-IR & $1.8(1.3)$ & $3.2(2.2)$ & $4.0(3.6)$ & 0.000 & 0.002 & 0.000 \\
\hline НОМА- $\beta$ & $110.7(76.0)$ & $95.7(64.5)$ & $60.9(60.0)$ & 0.003 & 0.000 & 0.000 \\
\hline RLP-C, mg/dL & $4.8(4.2)$ & $7.0(8.4)$ & $5.3(6.1)$ & 0.000 & 0.001 & 0.089 \\
\hline LDL-C, mg/dL & $117.7(39.2)$ & $118.1(39.5)$ & $108.4(29.7)$ & 0.934 & 0.003 & 0.000 \\
\hline calculated LDL-C, mg/dL & $117.4(41.1)$ & $113.5(36.2)$ & $99.0(28.1)$ & 0.521 & 0.000 & 0.000 \\
\hline sdLDL-C, mg/dL & $26.5(16.8)$ & $32.5(22.5)$ & $29.4(19.9)$ & 0.001 & 0.179 & 0.010 \\
\hline sdLDL-C/LDL-C ratio & $0.226(0.081)$ & $0.272(0.103)$ & $0.272(0.124)$ & 0.000 & 0.701 & 0.000 \\
\hline lbLDL-C, mg/dL & $89.8(27.4)$ & $84.4(22.7)$ & $76.4(24.1)$ & 0.115 & 0.000 & 0.000 \\
\hline LDL-TG, mg/dL & $20.1(9.9)$ & $23.3(10.6)$ & $19.3(7.9)$ & 0.000 & 0.000 & 0.194 \\
\hline Non HDL-C, mg/dL & $133.4(48.1)$ & $139.7(38.8)$ & $124.4(33.5)$ & 0.216 & 0.000 & 0.000 \\
\hline HDL-C, mg/dL & $59.9(18.8)$ & $53.6(16.7)$ & $50.3(17.2)$ & 0.000 & 0.061 & 0.000 \\
\hline $\mathrm{HDL}_{2}-\mathrm{C}, \mathrm{mg} / \mathrm{dL}$ & $34.7(16.1)$ & $29.8(13.7)$ & $28.4(11.8)$ & 0.000 & 0.479 & 0.000 \\
\hline
\end{tabular}

* Data are expressed as median (interquartile range).

** Data are expressed as number of obese participant (percentage of obese participants) $\left(\mathrm{BMI} \geq 27 \mathrm{~kg} / \mathrm{m}^{2}\right)$.

Apo, apolipoprotein; BMI, body mass index; BP, blood pressure; DM, diabetes mellitus; HbA1c, glycated hemoglobin; HOMA-IR, homeostatic model assessment on Insulin Resistance; HOMA- $\beta$, homeostatic model assessment on beta-cell; HDL-C, HDL cholesterol; lbLDL-C, large buoyant LDL cholesterol; LDL-C, LDL cholesterol; NGT, normal glucose tolerance; preDM, pre-diabetes mellitus; RLP-C, remnant lipoprotein cholesterol; sdLDL-C, small dense LDL cholesterol; TG, triglycerides

therapy, TG, RLP-C, sdLDL-C, sdLDL-C/LDL-C ratio, and LDL-TG values were all significantly higher than in normals, while HDL-C, HDL2-C, and apoE-HDL$\mathrm{C}$ were all significantly lower than in normal subjects (see Table 4). In female prediabetic subjects on statin therapy, TG, RLP-C, and sdLDL-C/LDL-C ratio values were all significantly higher than in normal, while HDL-C, HDL2-C, and apoE-HDL-C were all significantly lower than in normal (see Table 6).

In all male diabetic subjects, TG, sdLDL-C/LDL-C ratio, and LDL-TG values were all significantly higher than in normal subjects, while direct LDL-C, calculated LDL-C, non-HDL-C, HDL-C, HDL2-C, HDL3$\mathrm{C}$, and apoE-HDL-C were all significantly lower than in normal subjects (see Table 1). In diabetic men not on statin therapy, with very similar differences being observed in diabetic men not on statin therapy versus normal subjects (see Table 5). In all female diabetics, TG, RLP-C, sdLDL-C, and sdLDL-C/LDL-C ratio values were all significantly higher than in nortmal subjects, while lbLDL-C, HDL-C, and apoE-HDL-C were all significantly lower than in normal subjects. In all female diabetics off statin therapy, TG, sdLDL-C, and sdLDL-C/LDL-C ratio values were all significantly higher than in normal subjects, while direct LDL-C, calculated LDL-C, lbLDL-C, non-HDL-C, HDL-C, HDL2-C, HDL3-C, and apoE-HDL-C were all significantly lower than in normal subjects. Interestingly, median direct LDL-C values in male and female diabetic subjects in all three categories ranged from 104.1$108.4 \mathrm{mg} / \mathrm{dL}$, whereas for calculated LDL-C, these values ranged from $90.7-99.0 \mathrm{mg} / \mathrm{dl}$, with an average 
Table 5. Characteristics of male subjects on statins $(n=347)^{*}$

\begin{tabular}{|c|c|c|c|c|c|c|}
\hline \multirow[b]{2}{*}{ Variable } & \multirow{2}{*}{$\begin{array}{l}\text { NGT } \\
n=62\end{array}$} & \multirow{2}{*}{$\begin{array}{c}\text { PreDM } \\
n=79\end{array}$} & \multirow{2}{*}{$\begin{array}{c}\text { DM } \\
n=206\end{array}$} & \multicolumn{3}{|c|}{$P$ value } \\
\hline & & & & $\begin{array}{l}\text { NGT vs } \\
\text { PreDM }\end{array}$ & $\begin{array}{l}\text { PreDM } \\
\text { vs DM }\end{array}$ & $\begin{array}{c}\text { DM vs } \\
\text { NGT }\end{array}$ \\
\hline Age, year & $66.0(16.2)$ & $66.0(12.7)$ & $62.4(15.2)$ & 0.911 & 0.030 & 0.089 \\
\hline BMI, $\mathrm{kg} / \mathrm{m}^{2}$ & $24.7(3.4)$ & $25.0(4.1)$ & $26.6(4.9)$ & 0.923 & 0.000 & 0.001 \\
\hline Obesity Prevalence & $14(22.6 \%)^{* *}$ & $16(20.3 \%)^{* *}$ & $88(42.7 \%)^{* *}$ & 0.923 & 0.001 & 0.001 \\
\hline Systolic BP, mmHg & $130.0(15.3)$ & $127.0(12.0)$ & $130.0(10.0)$ & 0.630 & 0.797 & 0.694 \\
\hline Diastolic BP, mmHg & $75.0(10.8)$ & $75.0(11.0)$ & $70.0(10.0)$ & 0.742 & 0.878 & 0.447 \\
\hline Fasting Glucose, mg/dL & $94.0(7.0)$ & $106.0(8.5)$ & $132.0(39.8)$ & 0.000 & 0.000 & 0.000 \\
\hline HbAlc, \% & $5.6(0.3)$ & $5.9(0.4)$ & $6.8(1.4)$ & 0.000 & 0.000 & 0.000 \\
\hline Insulin, & $8.5(6.8)$ & $11.2(8.0)$ & $10.4(7.9)$ & 0.000 & 0.369 & 0.000 \\
\hline HOMA-IR & $1.9(1.5)$ & $3.0(2.1)$ & $3.5(3.0)$ & 0.000 & 0.081 & 0.000 \\
\hline НOMА- $\beta$ & $104.0(68.5)$ & $90.4(51.9)$ & $60.0(56.7)$ & 0.669 & 0.000 & 0.000 \\
\hline \multicolumn{7}{|l|}{ Lipids and Lipoproteins } \\
\hline Total cholesterol, mg/dL & $175.5(57.2)$ & $184.0(56.0)$ & $168.0(50.8)$ & 0.437 & 0.006 & 0.141 \\
\hline Triglycerides, mg/dL & $100.5(47.3)$ & $111.0(69.0)$ & $118.0(88.3)$ & 0.119 & 0.478 & 0.014 \\
\hline RLP-C, mg/dL & $5.7(5.6)$ & $7.4(8.3)$ & $7.3(9.4)$ & 0.066 & 0.779 & 0.096 \\
\hline LDL-C, mg/dL & $102.1(46.0)$ & $106.7(48.8)$ & $104.7(40.9)$ & 0.540 & 0.881 & 0.451 \\
\hline calculated LDL-C, mg/dL & $100.6(51.9)$ & $102.4(46.3)$ & $92.0(41.7)$ & 0.628 & 0.002 & 0.029 \\
\hline sdLDL-C, mg/dL & $27.5(15.0)$ & $33.4(22.4)$ & $33.2(21.0)$ & 0.070 & 0.847 & 0.016 \\
\hline sdLDL-C/LDL-C ratio & $0.265(0.070)$ & $0.288(0.121)$ & $0.304(0.140)$ & 0.054 & 0.486 & 0.003 \\
\hline lbLDL-C, mg/dL & $76.0(32.7)$ & $73.8(31.0)$ & $72.4(26.8)$ & 0.639 & 0.557 & 0.389 \\
\hline LDL-TG, mg/dL & $22.0(8.2)$ & $22.9(8.8)$ & $20.0(8.1)$ & 0.094 & 0.000 & 0.116 \\
\hline Non HDL-C, mg/dL & $122.9(51.7)$ & $131.1(50.3)$ & $119.5(44.6)$ & 0.260 & 0.013 & 0.404 \\
\hline HDL-C, mg/dL & $49.9(16.0)$ & $49.4(13.8)$ & $46.8(14.1)$ & 0.651 & 0.187 & 0.046 \\
\hline $\mathrm{HDL}_{2}-\mathrm{C}, \mathrm{mg} / \mathrm{dL}$ & $26.4(12.9)$ & $27.1(7.9)$ & $25.4(8.4)$ & 0.648 & 0.430 & 0.201 \\
\hline $\mathrm{HDL}_{3}-\mathrm{C}, \mathrm{mg} / \mathrm{dL}$ & $22.8(4.2)$ & $22.5(5.3)$ & $20.9(5.3)$ & 0.975 & 0.006 & 0.009 \\
\hline ApoE-HDL, mg/dL & $4.5(2.5)$ & $4.6(1.9)$ & $4.1(1.8)$ & 0.884 & 0.011 & 0.009 \\
\hline
\end{tabular}

* Data are expressed as median (interquartile range).

** Data are expressed as number of obese participant (percentage of obese participants) $\left(\mathrm{BMI} \geq 27 \mathrm{~kg} / \mathrm{m}^{2}\right)$.

Apo, apolipoprotein; BMI, body mass index; BP, blood pressure; DM, diabetes mellitus; HbA1c, glycated hemoglobin; HOMA-IR, homeostatic model assessment on Insulin Resistance; HOMA- $\beta$, homeostatic model assessment on beta-cell; HDL-C, HDL cholesterol; lbLDL-C, large buoyant LDL cholesterol; LDL-C, LDL cholesterol; NGT, normal glucose tolerance; preDM, pre-diabetes mellitus; RLP-C, remnant lipoprotein cholesterol; sdLDL-C, small dense LDL cholesterol; TG, triglycerides

underestimation of $11.5 \%(p<0.001)$.

For all groups, we have tabulated relative percent differences between normal, prediabetic, and diabetic male and female subjects for all lipid and lipoprotein subfractions (see Figs.1, 2, and 3). A number of different patterns emerged. One pattern is that prediabetic subjects had the greatest increases in RLP-C, sdLDL-C, and LDL-TG values, especially in female subjects, as compared with normal subjects. Another pattern that emerged is that diabetic subjects had the greatest decreases in HDL-C and its subfractions as compared with normal, especially in men. In women, significant decreases in HDL-C and its subfractions were observed in prediabetic subjects as compared with normal; however, this was not the case for prediabetic men. A final pattern that emerged was that calculated
LDL-C significantly underestimated direct LDL-C values in diabetic male and female subjects, with less discrepancy observed in normal and prediabetic subjects.

We examined the effects of obesity classified as having a BMI $>27 \mathrm{~kg} / \mathrm{m}^{2}$ versus being non-obese in normal, prediabetic, and diabetic men and women. The data are shown in Tables 7 and 8. In normal men, being obese resulted in significantly higher systolic blood pressure, insulin, HOMAIR, $\mathrm{HOMA}_{\beta}$, TG, sdLDL-C, sdLDL-C/LDL-C ratio, non-HDL-C, and LDL-TG values, and significantly lower HDL-C, HDL2-C, and apoE-HDL-C as compared with non-obese normal subjects (see Table 7). In normal women, being obese resulted in the same differences except that TG and non-HDL-C levels were not significantly higher, but HDL3-C was significantly lower as compared with 
Table 6. Characteristics of female subjects on statins $(n=339) *$

\begin{tabular}{|c|c|c|c|c|c|c|}
\hline \multirow[b]{2}{*}{ Variable } & \multirow{2}{*}{$\begin{array}{l}\text { NGT } \\
n=60\end{array}$} & \multirow{2}{*}{$\begin{array}{c}\text { PreDM } \\
n=52\end{array}$} & \multirow{2}{*}{$\begin{array}{c}\mathrm{DM} \\
n=227\end{array}$} & \multicolumn{3}{|c|}{$P$ value } \\
\hline & & & & $\begin{array}{l}\text { NGT vs } \\
\text { PreDM }\end{array}$ & $\begin{array}{l}\text { PreDM } \\
\text { vs DM }\end{array}$ & $\begin{array}{c}\text { DM vs } \\
\text { NGT }\end{array}$ \\
\hline Age, year & $69.5(13.0)$ & $68.0(12.8)$ & $64.3(13.1)$ & 0.972 & 0.012 & 0.011 \\
\hline BMI, $\mathrm{kg} / \mathrm{m}^{2}$ & $24.0(4.1)$ & $23.5(4.5)$ & $26.2(5.4)$ & 0.297 & 0.000 & 0.000 \\
\hline Obesity Prevalence & $10(16.7 \%)^{* *}$ & $8(15.4 \%)^{* *}$ & $94(41.4 \%)^{* *}$ & 0.297 & 0.001 & 0.001 \\
\hline Systolic BP, mmHg & $129.5(18.3)$ & $130.0(10.0)$ & $130.0(10.0)$ & 0.793 & 0.840 & 0.814 \\
\hline Diastolic BP, mmHg & $76.0(10.5)$ & $77.0(12.0)$ & $70.0(10.0)$ & 0.840 & 0.602 & 0.452 \\
\hline Fasting Glucose, mg/dL & $92.0(8.5)$ & $104.5(10.3)$ & $131.0(42.0)$ & 0.000 & 0.000 & 0.000 \\
\hline HbAlc, \% & $5.6(0.5)$ & $5.9(0.4)$ & $6.9(1.3)$ & 0.001 & 0.000 & 0.000 \\
\hline Insulin, & $10.1(7.6)$ & $13.4(10.2)$ & $13.7(10.5)$ & 0.009 & 0.738 & 0.000 \\
\hline HOMA-IR & $2.3(1.7)$ & $3.4(2.6)$ & $4.6(3.9)$ & 0.000 & 0.005 & 0.000 \\
\hline HOMA- $\beta$ & $128.9(85.5)$ & $116.4(75.1)$ & $69.2(72.7)$ & 0.097 & 0.000 & 0.000 \\
\hline \multicolumn{7}{|l|}{ Lipids and Lipoproteins } \\
\hline Total cholesterol, mg/dL & $193.8(56.0)$ & $193.5(54.5)$ & $172.0(41.0)$ & 0.433 & 0.007 & 0.000 \\
\hline Triglycerides, mg/dL & $107.6(68.3)$ & $131.5(106.3)$ & $120.0(78.0)$ & 0.003 & 0.038 & 0.162 \\
\hline $\mathrm{RLP}-\mathrm{C}, \mathrm{mg} / \mathrm{dL}$ & $7.4(5.8)$ & $9.5(14.6)$ & $6.8(8.7)$ & 0.012 & 0.003 & 0.809 \\
\hline LDL-C, mg/dL & $112.5(40.6)$ & $110.0(49.0)$ & $104.1(39.6)$ & 0.733 & 0.490 & 0.142 \\
\hline calculated LDL-C, mg/dL & $111.2(52.1)$ & $102.9(55.1)$ & $90.7(35.4)$ & 0.261 & 0.015 & 0.000 \\
\hline sdLDL-C, mg/dL & $30.0(17.2)$ & $35.8(21.4)$ & $32.8(16.9)$ & 0.077 & 0.181 & 0.371 \\
\hline sdLDL-C/LDL-C ratio & $0.278(0.091)$ & $0.330(0.136)$ & $0.301(0.131)$ & 0.003 & 0.255 & 0.007 \\
\hline $\mathrm{lbLDL}-\mathrm{C}, \mathrm{mg} / \mathrm{dL}$ & $81.7(31.4)$ & $72.2(29.5)$ & $71.7(27.8)$ & 0.067 & 0.926 & 0.012 \\
\hline LDL-TG, mg/dL & $23.8(6.1)$ & $26.9(10.1)$ & $21.7(8.6)$ & 0.105 & 0.000 & 0.015 \\
\hline Non HDL-C, mg/dL & $135.9(53.9)$ & $138.5(54.9)$ & $117.3(36.8)$ & 0.972 & 0.002 & 0.001 \\
\hline HDL-C, mg/dL & $58.2(15.4)$ & $51.0(12.6)$ & $54.0(13.9)$ & 0.002 & 0.044 & 0.042 \\
\hline $\mathrm{HDL}_{2}-\mathrm{C}, \mathrm{mg} / \mathrm{dL}$ & $32.1(11.1)$ & $26.1(9.1)$ & $30.3(10.2)$ & 0.002 & 0.003 & 0.152 \\
\hline $\mathrm{HDL}_{3}-\mathrm{C}, \mathrm{mg} / \mathrm{dL}$ & $24.9(3.9)$ & $22.8(4.9)$ & $23.4(5.3)$ & 0.092 & 0.875 & 0.025 \\
\hline ApoE-HDL, mg/dL & $5.8(2.0)$ & $4.9(1.4)$ & $5.0(1.8)$ & 0.007 & 0.606 & 0.003 \\
\hline
\end{tabular}

* Data are expressed as median (interquartile range).

** Data are expressed as number of obese participant (percentage of obese participants) $\left(\mathrm{BMI} \geq 27 \mathrm{~kg} / \mathrm{m}^{2}\right)$.

Apo, apolipoprotein; BMI, body mass index; BP, blood pressure; DM, diabetes mellitus; HbA1c, glycated hemoglobin; HOMA-IR, homeostatic model assessment on Insulin Resistance; HOMA- $\beta$, homeostatic model assessment on beta-cell; HDL-C, HDL cholesterol; lbLDL-C, large buoyant LDL cholesterol; LDL-C, LDL cholesterol; NGT, normal glucose tolerance; preDM, pre-diabetes mellitus; RLP-C, remnant lipoprotein cholesterol; sdLDL-C, small dense LDL cholesterol; TG, triglycerides

normal subjects (see Table 8). In prediabetic men, being obese resulted in the same findings as for normal men, except that no significant differences with regard to HDL-C and its subfractions were noted as compared with non-obese prediabetic men. In prediabetic women, being obese resulted in only significantly higher systolic blood pressure, HbA1c, insulin, HOMAIr, HOMA $\beta$, but no other parameters, as compared with prediabetic, non-obese women (see Table 8). In diabetic men, being obese resulted in significantly higher insulin, HOMAIR, $\mathrm{HOMA}_{\beta}$, TG, RLP-C, sdLDL-C, sdLDL-C/LDL-C ratio, LDL-TG, lbLDL-C, non-HDL-C, and significantly lower levels of HDL-C, HDL2-C, HDL3-C, and apoE-HDL-C, compared with non-obese diabetic men (see Table 7). In contrast, in diabetic women, being obese was only associated with significantly higher lev- els of insulin and $\mathrm{HOMA}_{\beta}$, and significantly lower levels of HDL2-C, as compared with non-obese diabetic women (see Table 8). Surprisingly, in this analysis, the presence of obesity had little effect on fasting glucose levels, possibly because group selections by gender were based on this parameter. Similar effects of obesity using the $\geq 25 \mathrm{~kg} / \mathrm{m}^{2}$ criteria were observed in all of the above subgroups (see Supplementary Tables 1 and 2). It should also be noted that, as previously mentioned, the prevalence of obesity was significantly higher in prediabetic and diabetic men and women versus controls as shown in Tables 1 and 2 .

We also examined the effects of statin use in normal men and women (see Supplementary Table 3), in prediabetic men and women (see Supplementary Table 4), and in diabetic men and women (see Sup- 
(A)

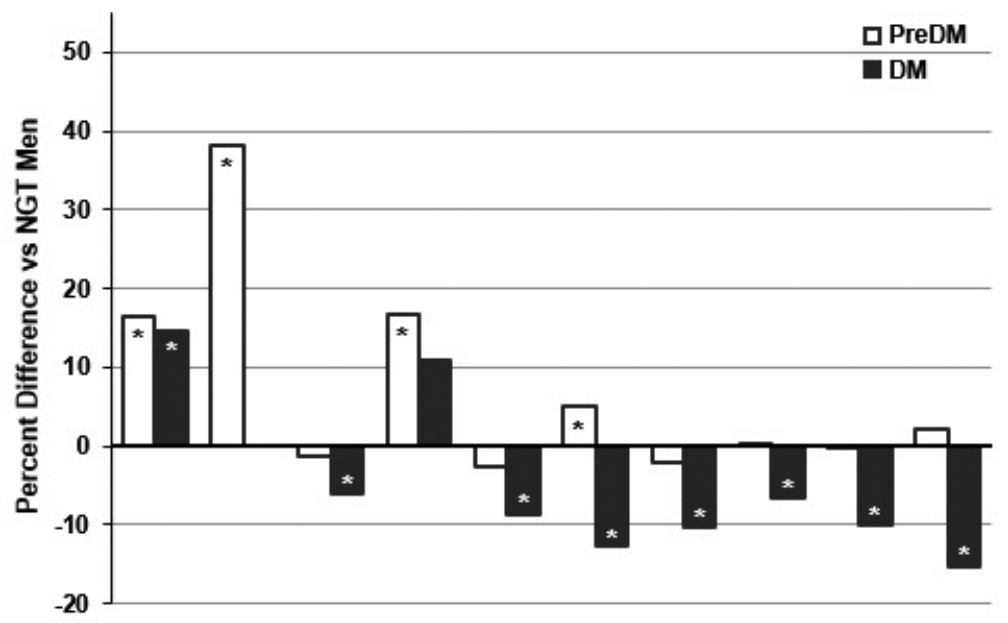

(B)

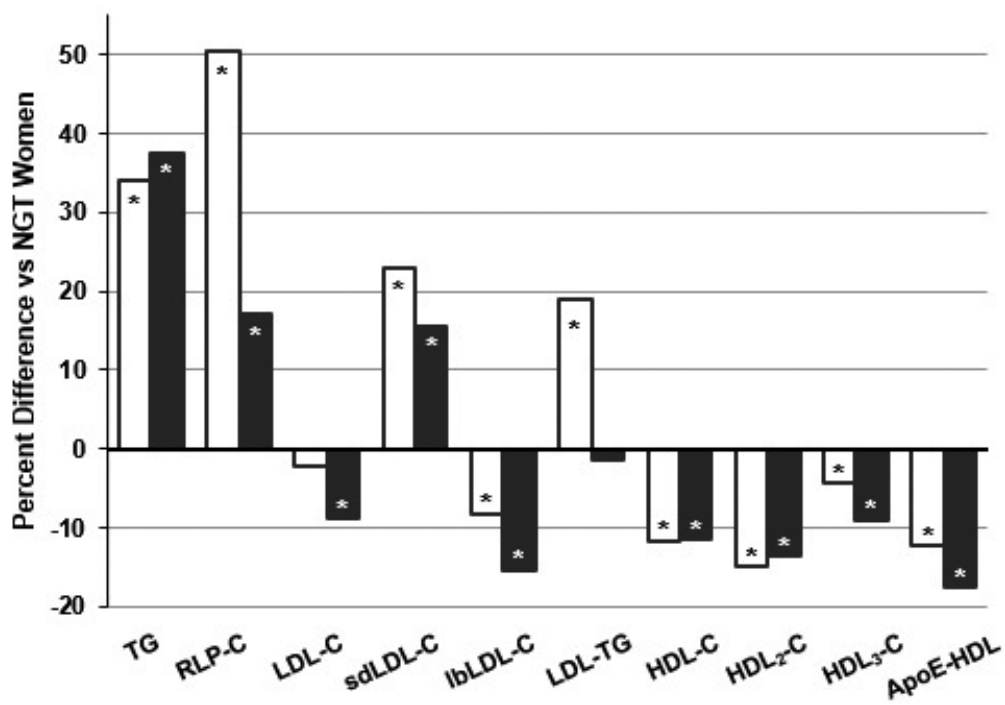

Fig. 1. (A) Percent differences of lipid variables in all male subjects with prediabetes (PreDM; white column) or diabetes (DM; black column) from non-diabetic. (B) Percent differences of lipid variables in all female subjects with prediabetes (PreDM; white column) or diabetes (DM; black column) from non-diabetic. Star sign $\left(^{*}\right)$ indicate $p<0.05$

plementary Table 5). In normal men, statin use was significantly associated with being older, having a higher BMI, and having a higher prevalence of obesity as compared with non-users. In prediabetic men, statin use was significantly associated with being older and having lower RLP-C values than non-users. In diabetic men, statin use was significantly associated with higher BMI, obesity prevalence, LDL-TG, and apoEHDL-C values as compared with non-users. In normal women, statin use was associated with significantly higher age, BMI, obesity prevalence, systolic blood pressure, diastolic blood pressure, glucose, $\mathrm{HbA1c}$, insulin, HOMAIR, TG, lbLDL-C, and sdLDL/LDL-C ratio as compared with non-users. In prediabetic women, statin use was significantly associated with higher age, systolic blood pressure, TG, RLP-C, sdLDL/LDL-C ratio, and LDL-TG as compared with non-users. In diabetic women, statin use was significantly associated with higher BMI, sdLDL/LDL-C ratio, LDL-TG, HDL-C, HDL3-C, and apoE-HDL-C as compared with non-users.

We have also examined correlations between parameters in men and women participating in this study (see Supplementary Tables 6, 7, and 8). We observed very strong correlations between TC, calculated LDLC, direct LDL-C, sdLDL-C, and LDL-TG, and somewhat weaker correlations between TC and HDL3-C, HDL-C, apoE HDL-C, and RLP-C. The strongest 
(A)

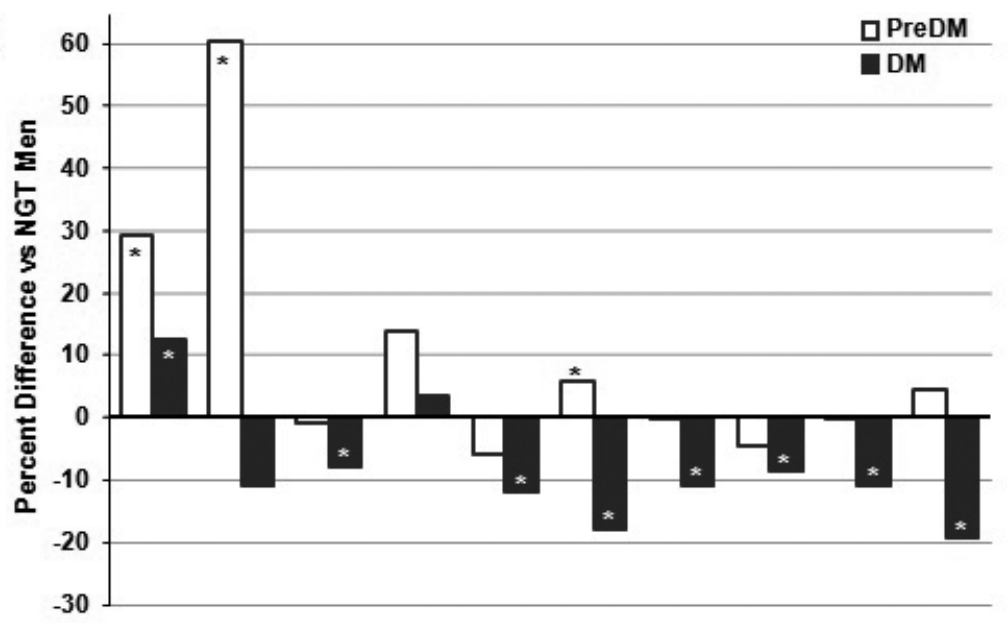

(B)

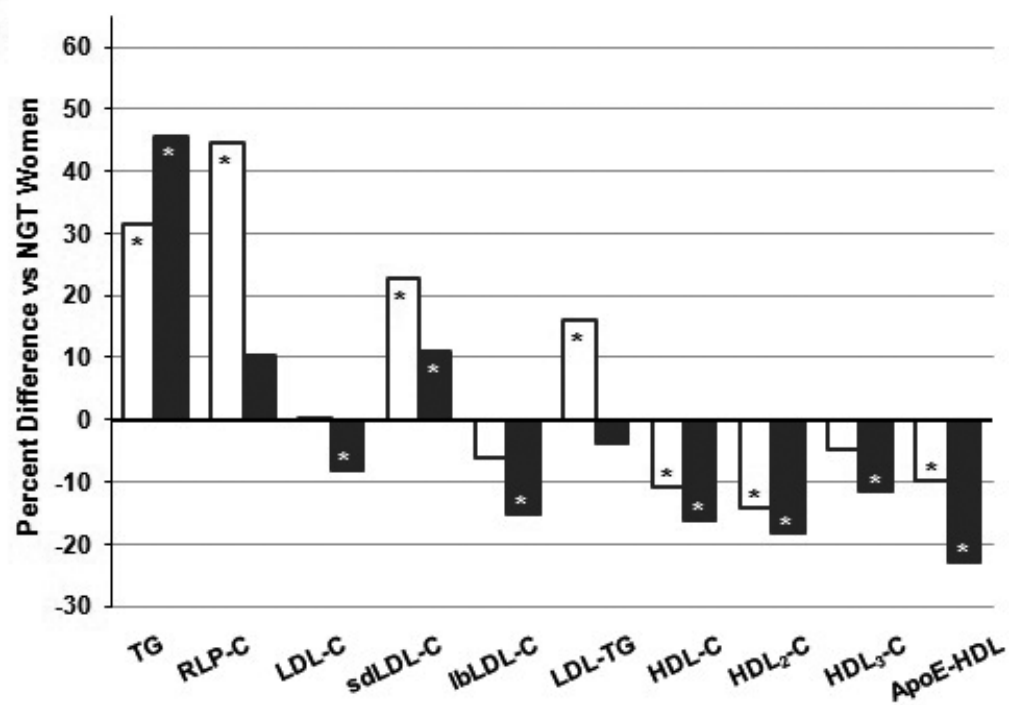

Fig.2. (A) Percent differences of lipid variables in male subjects without lipid medication with prediabetes (PreDM; white column) or diabetes (DM; black column) from non-diabetic. (B) Percent differences of lipid variables in all female subjects without lipid medication with prediabetes (PreDM; white column) or diabetes (DM; black column) from nondiabetic. Star sign $(*)$ indicate $p<0.05$

positive correlations with TG levels were with RLP-C, LDL-TG, and sdLDL-C, as well as strong inverse correlation with HDL-C and somewhat weaker correlations with HDL3-C and apoE HDL-C. We also noted that insulin and HOMAIR values were significantly correlated with TG values, while НОМАв was modestly correlated with LDL-TG.

\section{Discussion}

In order to prevent CVD in prediabetic and diabetic subjects, not only glycemic control, but control of blood pressure and lipids and their subfractions are also important. Our focus in this study was to carefully examine lipids and lipoprotein subfractions using standard and novel markers in subjects with prediabetes and diabetes as compared with normal subjects in a large Asian population of over 2,000 individuals. In addition, we examined these parameters in both men and women, as well as in those who are off and on lipid-lowering medication. In addition, we related these parameters to markers of glucose homeostasis, namely fasting values of glucose, insulin, $\mathrm{HbAlc}$, and calculated HOMAir and $\mathrm{HOMA}_{\beta}$. Our data reinforces the concept that both increased insulin resistance and decreased insulin production underlie the pathogene- 
(A)

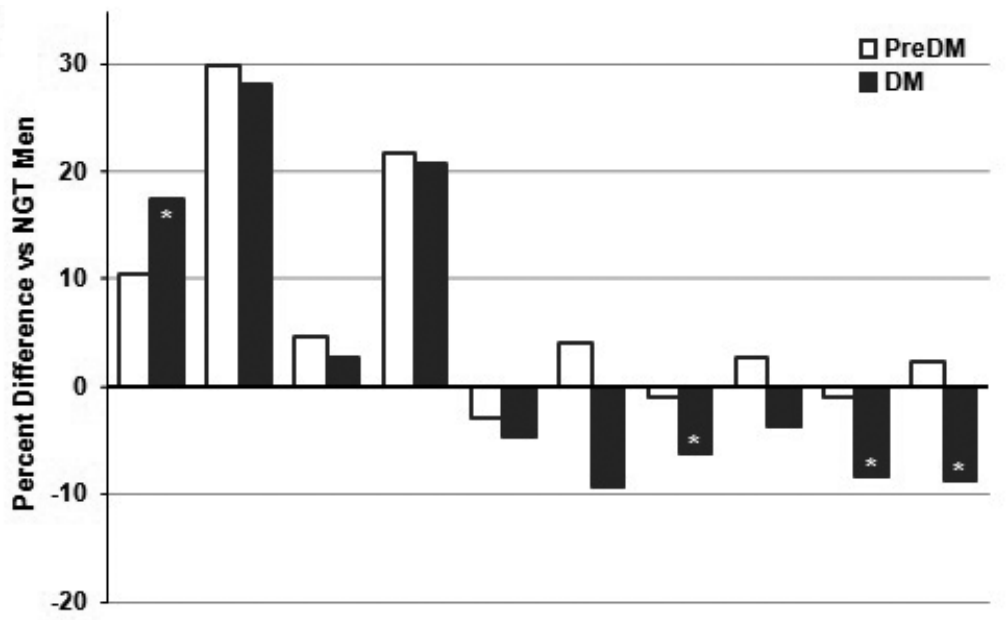

(B)

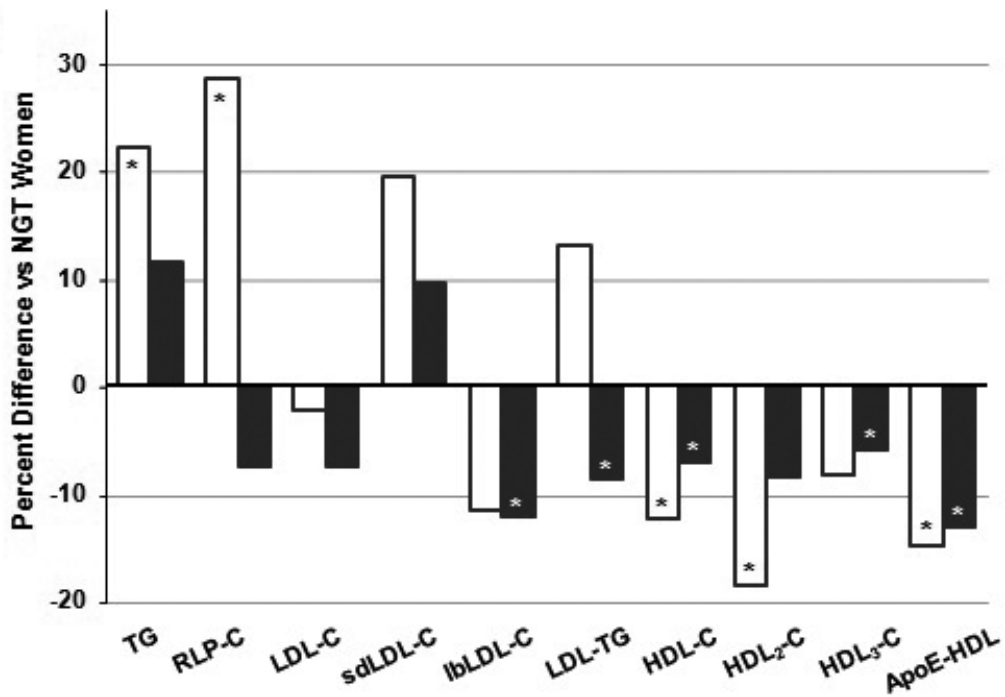

Fig.3. (A) Percent differences of lipid variables in male subjects on statin treatment with prediabetes (PreDM; white column) or diabetes (DM; black column) from non-diabetic. (B) Percent differences of lipid variables in all female subjects on lipid medication with prediabetes (PreDM; white column) or diabetes (DM; black column) from non-diabetic. Star sign $\left.{ }^{*}\right)$ indicate $p<0.05$

sis of prediabetes and the transition to diabetes, and that obesity plays an important role in this process. Recent studies using novel automated assays indicate that elevated levels of sdLDL-C and LDL-TG provide significant information about CVD risk above and beyond standard risk factors, and this may be the case for decreased levels of HDL subfractions and apoE HDL-C as well ${ }^{27-33,46)}$. However, to our knowledge, such new assays have not been applied to a relatively large population of male and female prediabetic and diabetic subjects.

It has long been known that diabetics have dyslipidemia with elevated TG, RLP-C, sdLDL-C, and decreased HDL-C values as compared to controls ${ }^{1-4)}$. However, these older observations were made with a more manual cumbersome method of measuring RLP-C and gradient gel technology for assessing LDL particle size. In addition, more recently, it has been documented that diabetic subjects on statin therapy get additional benefit from the addition of fenofibrate if they have fasting TG values $>204 \mathrm{mg} / \mathrm{dL}$ and HDL-C values $<35 \mathrm{mg} / \mathrm{dL}^{17}{ }^{19)}$. It is also known that statins can have beneficial effects not only on calculated LDL-C, but also on TG, RLP-C, direct LDL-C, sdLDL-C, and sdLDL-C ${ }^{39)}$. While statins clearly have strikingly beneficial effects on CVD risk reduction in 


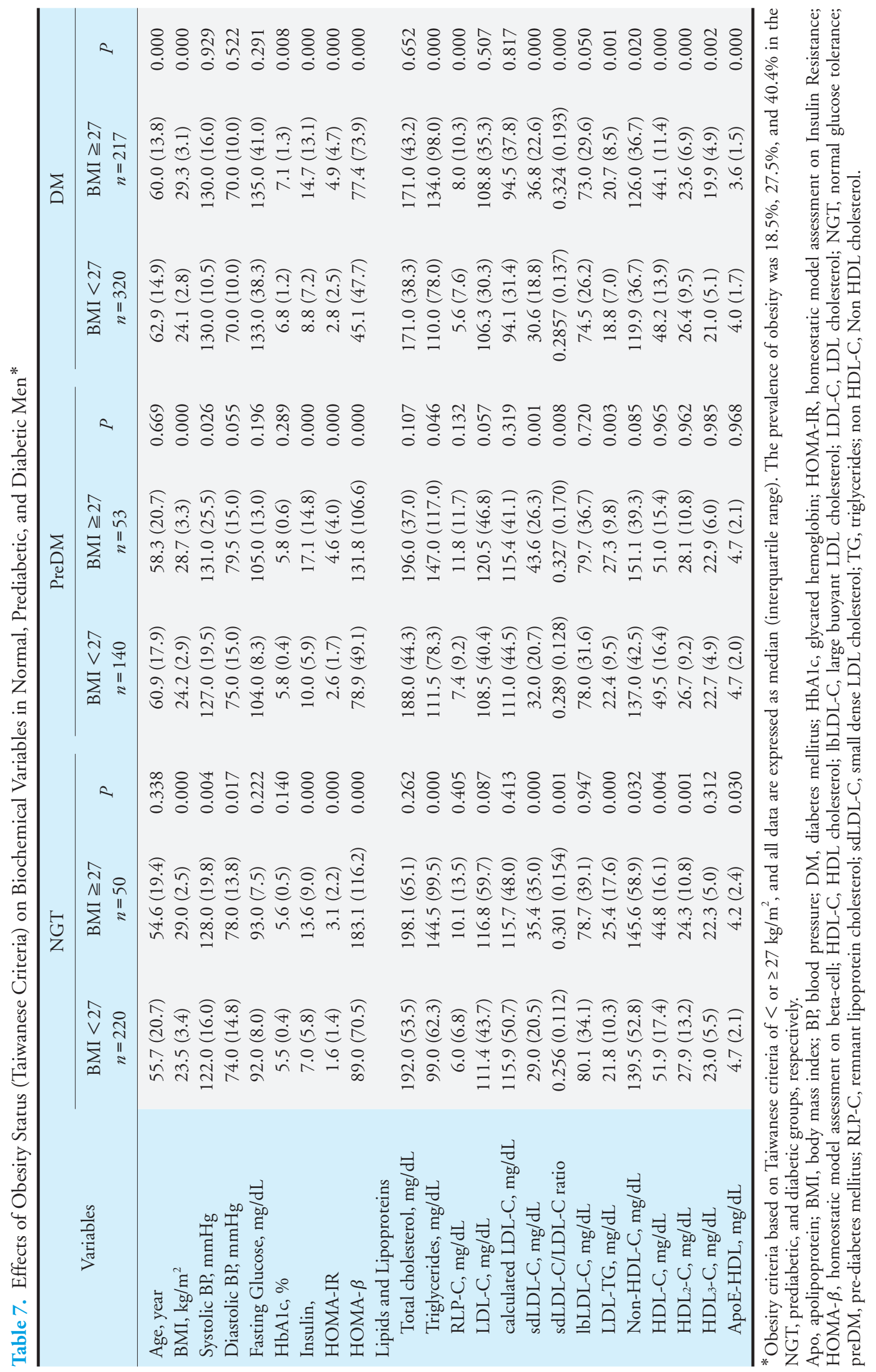




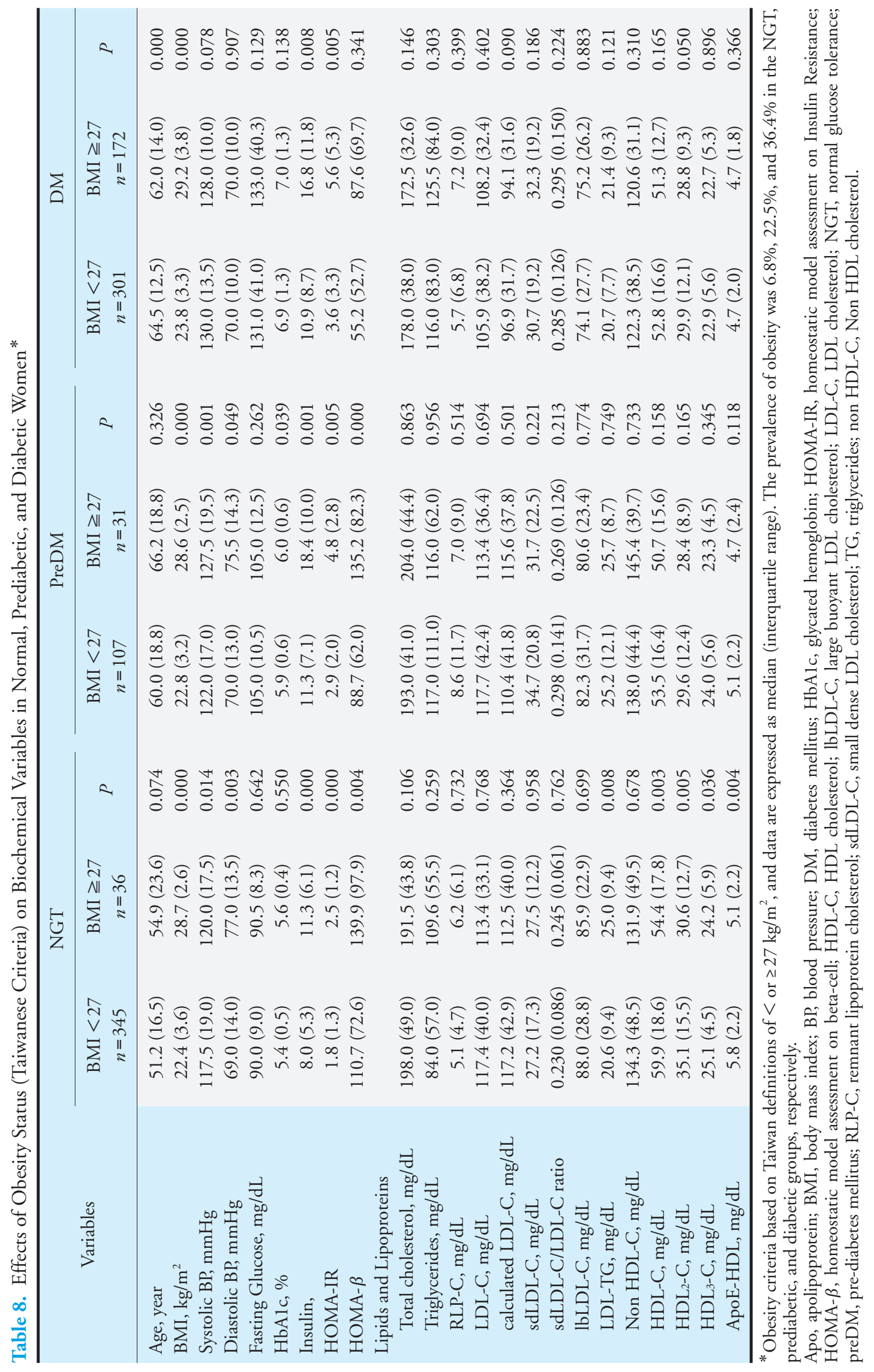


prediabetics and diabetics, there is substantial residual risk seen in these subjects on statin treatment ${ }^{19)}$.

In the present study, fasting triglycerides were significantly higher in male and female prediabetic and diabetic subjects as compared with controls in all subjects, as well as in those on and off lipid-lowering medications, except for male prediabetics and female diabetics on lipid-lowering therapy. RLP-C levels were highest in prediabetics, and in this group, lipid-lowering treatment abolished these differences in men, but not in women. It is known that RLP-C may be an especially important CVD risk factor in women, and it is possible that the addition of fibrates or omega-3 fatty acids on top of statin therapy may be very important to minimize CVD risk ${ }^{46)}$. With regard to direct LDL-C, calculated LDL-C, and lbLDL-C differences between prediabetes and diabetics versus control subjects were relatively small. An important novel finding in this study was that calculated LDL-C, using the Friedewald formula, significantly underestimated direct LDL-C with the assay used by slightly more than $10 \%$. We therefore recommend the use of direct LDL-C in this population because it is the primary target of lipid-lowering therapy.

It should also be noted that sdLDL has been shown to be the more atherogenic component of LDL. Prediabetic subjects were most likely to have increases in this parameter, as compared with control subjects. However, sdLDL-C differences were greater in female prediabetic and diabetic subjects than in men, and the use of statin therapy in these subjects did not abolish differences in women. These finding indicate that possibly, either more intensive statin therapy or additional treatments such as fibrates or omega-3 fatty acids may be necessary to treat elevated TG-rich lipoproteins and sdLDL-C in prediabetic and diabetic subjects to minimize residual CVD risk ${ }^{47)}$.

LDL-TG, a new CVD marker, was highest in the prediabetic subjects, and statin therapy did not minimize these differences. Since a recent study has shown that LDL-TG levels are higher in CHD than in controls, our findings may be one of the reasons why prediabetes is linked to atherosclerosis ${ }^{32)}$. However, it was unexpected that LDL-TG levels were lower in this population in diabetic subjects than in normal subjects. We have observed a modest positive correlation between $\mathrm{HOMA}_{\beta}$ and LDL-TG, and since diabetics have lower $\mathrm{HOMA}_{\beta}$ values, this may account for this difference. Whether this finding is also true in other populations remains to be confirmed. It should be noted that insulin deficiency and decreased $\mathrm{HOMA}_{\beta}$ appears to be more common among Asian diabetics than that observed in western Caucasian populations where obesity and insulin resistance may be more common ${ }^{47,48)}$. This may also explain why we see differences in LDL-TG values in this population between prediabetic and diabetic subjects.

In addition, direct LDL-C levels were also lower in diabetic subjects, however, sdLDL-C levels were higher or similar to controls, resulting in sdLDL-C/ LDL-C ratios in patients with T2DM that were significantly higher than in any other group. A previous study in a Japanese population has assessed cholesterol and TG in twenty lipoprotein subfractions, and documented that smaller LDL particles carry less TG than larger LDL and VLDL particles ${ }^{49)}$. From this viewpoint, it makes sense that LDL-TG levels are lower in diabetics than in controls, since the number of sdLDL particles increases in patients with diabetes. Our overall data indicate that attempts to normalize sdLDL-C and LDL-TG levels in prediabetic and diabetic subjects need to be made to normalize their CVD risk.

Diabetics in our population and female prediabetics had significantly lower levels of HDL-C and HDL subfractions than controls, placing them at increased CVD risk. We have observed significant correlations between HDL-C, HDL3-C, HDL2-C, and apoE-HDL$\mathrm{C}$, and noted significantly lower values in diabetic men and prediabetic and diabetic women as compared to controls. These differences persisted for all parameters in patients on lipid-lowering treatment, except for $\mathrm{HDL}_{2}-\mathrm{C}$. It is still uncertain whether apoE-rich HDL particles are athero-protective. Similarly, there is an ongoing debate as to whether serum $\mathrm{HDL}_{3}-\mathrm{C}$ is cardioprotective. While serum glucose levels in prediabetes are not high enough to be diagnosed as diabetes, overall, their lipid values are similar to those in diabetics. Our findings on the pattern of lipid profiles support the concept that CVD risk in prediabetics may be as high as in those with diabetes.

The strength of this study is the relatively large number of participants in the 3 groups for both genders. Moreover, we examined subjects on and off statin therapy. The data trends were very similar in all groups. In addition, all subjects were on stable therapy for their lipid alterations as well as for diabetes if present for at least 6 months prior to the study. While absolute values of lipid parameters are known to be influenced by lipid medications, the trends and differences observed when normal, prediabetics, and diabetics were compared, was not greatly different. The participants with diabetes were also taking anti-diabetes medications, mostly metformin, but all subjects receiving insulin were excluded. Metformin and other glucose-lowering medication may affect lipid profiles, but do not cause rapid changes since their diabetic conditions were stable and their medications were not changed for at least 6 months. While we only studied a Tai- 
wanese population, another limitation of this study, these findings need to be replicated in other populations using these newer lipoprotein assays. It should be noted that our study was cross-sectional, and prospective treatment studies in diabetics or prediabetics need to be performed on order to further assess potential changes in the various lipoprotein fractions measured.

\section{Conclusion}

In summary, we compared novel lipoprotein parameters in normal, prediabetic and diabetic subjects. Prediabetic subjects were more likely to have elevated TG, RLP-C, sdLDL-C, and LDL-TG, while diabetic subjects were more likely to have decreases in HDL-C and HDL subfractions than normal subjects. These findings indicate that in this large Asian population, prediabetes is associated with greater increases in atherogenic lipoproteins, while diabetes is more likely to be associated with low levels of HDL and its subfractions. These findings suggest that insulin resistance, as seen in prediabetics, plays an important role in modulating changes TG-rich lipoproteins, while both insulin resistance and impaired insulin production, as seen in the diabetic state, may be more important in the regulation of HDL and its subfractions. In our view, both increases in atherogenic lipoproteins and decreases in HDL and its subfractions modulates CVD risk. Weight loss may be the best strategy to improve these abnormalities, and in the case of atherogenic lipoproteins, the combination of statins, fibrates, and omega-3 fatty acids. Moreover, our data indicate that calculated LDL-C significantly underestimated direct LDL-C in subjects with diabetes. In our view, direct LDL-C and sdLDL-C should be measured and optimized in both diabetic and prediabetic subjects to reduce CVD risk.

\section{Acknowledgements}

The contribution of all participants in this study is gratefully acknowledged and gratefully appreciated. Also, the authors thank Dr. Margaret Diffenderfer for assistance in manuscript preparation.

\section{Funding}

This study was supported by the research funding of the Lotung Poh-ai Hospital, Lotung, Taiwan. The research project reference AEP2017070059

\section{Conflict of Interests}

YI is an employee of Denka-Seiken Corporation,
MA is supported by a research grant from DenkaSeiken Co., Tokyo, Japan. All other authors declare no potential conflicts of interest with respect to the research, authorship, and/or publication of this article.

\section{Ethical Approval}

The research was approved by the ethics committee of the School of Medicine, Tokyo Medical and Dental University, Tokyo, Japan. (Reference number: M2000-2350).

\section{Contributorship}

$\mathrm{HH}$ and MA designed and conducted the study. $\mathrm{HH}, \mathrm{PH}$, and $\mathrm{MHC}$ recruited study subjects and collected data. $\mathrm{HH}, \mathrm{EK}$, and MA, analyzed the data. $\mathrm{HH}$, MA, and EJS wrote the first version of the manuscript. All authors reviewed and edited the manuscript and approved the final version of the manuscript.

\section{References}

1) Siegel RD, Cupples A, Schaefer EJ, and Wilson PW: Apolipoproteins, and low-density lipoprotein size among diabetics in the Framingham offspring study, Metabolism. 1996; 45: 1267-1272

2) Ai M, Tanaka A, Ogita K, Sekinc M, Numano F, Numano F, and Reaven GM: Relationship between plasma insulin concentration and plasma remnant lipoprotein response to an oral fat load in patients with type 2 diabetes, J Am Coll Cardiol. 2001; 38: 1628-1632

3) Schaefer EJ, McNamara JR, Shah PK, Nakajima K, Cupples LA, Ordovas JM, and Wilson PW: Elevated remnantlike particle cholesterol and triglyceride levels in diabetic men and women in the Framingham Offspring Study. Diabetes Care, 2002; 25: 989-994

4) Hirano T, Pathophysiology of Diabetic Dyslipidemia. J Atheroscler Thromb. 2018; 25: 771-782

5) Brannick B, and Dagogo-Jack S, Prediabetes and cardiovascular disease: pathophysiology and interventions for prevention and risk reduction. Endocrinol Metab Clin North Am. 2018; 47: 33-50

6) Turner RC, Millns H, Neil HAW, Stratton IM, Manley SE, Matthews DR, and Holman RR: Risk factors for coronary artery disease in non-insulin dependent diabetes mellitus: United Kingdom Prospective Diabetes Study (UKPDS: 23). BMJ. 1998; 316: 823-828

7) Pyörälä K, Pedersen TR, Kjekshus J, Faergeman O, Olsson AG, and Thorgeirsson G: Cholesterol lowering with simvastatin improves prognosis of diabetic patients with coronary heart disease: a subgroup analysis of the Scandinavian Simvastatin Survival Study (4S). Diabetes Care. 1997; 20: 614-620

8) Goldberg RB, Mellies MJ, Sacks FM, Moyé LA, Howard BV, Howard WJ, Davis BR, Cole TG, Pfeffer MA, and Braunwald E: Cardiovascular events and their reduction with pravastatin in diabetic and glucose-intolerant myo- 
cardial infarction survivors with average cholesterol levels: subgroup analyses in the cholesterol and recurrent events (CARE) trial. Circulation. 1998; 98: 2513-2519

9) Keech A, Colquhoun D, Best J, Kirby A, Simes RJ, Hunt D, Hague W, Beller E, Arulchelvam M, Baker J, and Tonkin A: Secondary prevention of cardiovascular events with long-term pravastatin in patients with diabetes or impaired fasting glucose: results from the LIPID trial. Diabetes Care. 2003; 26: 2713-2721

10) Collins R, Armitage J, Parish S, Sleigh P, and Peto R: MRC/BHF Heart Protection Study of cholesterol-lowering with simvastatin in 5963 people with diabetes: a randomised placebo-controlled trial. Lancet. 2003; 361: 20052016

11) Colhoun HM, Betteridge DJ, Durrington PN, Hitman GA, Neil HAW, Livingstone SJ, Thomason MJ, Mackness MI, Charlton-Menys V, Fuller JH, and CARDS Investigators: Primary prevention of cardiovascular disease with atorvastatin in type 2 diabetes in the Collaborative Atorvastatin Diabetes Study (CARDS): multicentre randomised placebo-controlled trial. Lancet. 2004; 364: 685-696

12) Goff DC, Lloyd-Jones DM, Bennett G, Coady S, D'agostino RB, Gibbons R, Greenland P, Lackland DT, Levy D, O’Donnel CJ, Robinson JG, Schwartz JS, Shero ST, Smith SC, Sorlie P, Stone NJ, and Wilson PWF: 2013 ACC/ AHA guideline on the assessment of cardiovascular risk: a report of the American College of Cardiology/American Heart Association Task Force on Practice Guidelines. J Am Coll Cardiol. 2014; 63: 2935-2959

13) Stone NJ, Robinson JG, Lichtenstein AH, Merz CNB, Blum CB, Eckel RH, Goldberg AC, Gordon D, Levy D, Lloyd-Jones DM, McBride P, Schwartz JS, Shero ST, Smith SC, Watson K, and Wilson PWF: 2013 ACC/AHA guideline on the treatment of blood cholesterol to reduce atherosclerotic cardiovascular risk in adults: a report of the American College of Cardiology/American Heart Association Task Force on Practice Guidelines. J Am Coll Cardiol. 2014; 63: 2889-2934

14) American Diabetes Association. Cardiovascular disease and risk management: standards of medical care in diabetes - 2018. Diabetes Care. 2018; 41: S86-S104

15) Silverman MG, Ference BA, Im K, Wiviott SD, Giugliano RP, Grundy SM, Braunwald E, and Sabatine MS: Association between lowering LDL-C and cardiovascular risk reduction among different therapeutic interventions: a systematic review and meta-analysis. JAMA. 2016; 316: 1289-1297

16) Lloyd-Jones DM, Morris PB, Ballantyne CM, Birtcher KK, Daly DD, DePalma SM, Minissian MB, Orringer CE, SC Smith and Writing Committee: 2016 ACC expert consensus decision pathway on the role of non-statin therapies for LDL-cholesterol lowering in the management of atherosclerotic cardiovascular disease risk: a report of the American College of Cardiology Task Force on Clinical Expert Consensus Documents. J Am Coll Cardiol. 2016; 68: 92-125

17) DAROC Clinical Practice Guidelines for Diabetes Care2018, Taiwan, Diabetes Association of the ROC, 2018

18) Taiwan National Health Insurance Medication Prescription Guidelines- 2018, Section 2 - Cardiovascular-renal drugs, Taiwan, Ministry of Health and Welfare, National
Health Insurance Administration

19) Teramoto T, Sasaki J, Ishibashi S, Birou S, Daida H, Dohi S, Egusa G, Hiro T, Hirobe K, Iida M, Kihara S, Kinoshita M, Maruyama C, Ohta T, Okamura T, Yamashita S, Yokode M, and Yokote K: Executive summary of the Japan Atherosclerosis Society (JAS) guidelines for the diagnosis and prevention of atherosclerotic cardiovascular diseases in Japan - 2012 version. J Atheroscler Thromb. 2013; 20: 517-523

20) ACCORD Study Group. Effects of combination lipid therapy in type 2 diabetes mellitus. N Engl J Med. 2010; 362: 1563-1574

21) Austin MA, Breslow JL, Hennekens CH, Buring JE, Willett WC, and Krauss RM: Low-density lipoprotein subclass patterns and risk of myocardial infarction. JAMA. 1988; 260: 1917-1921

22) Campos H, Genest JJ, Blijlevens E, McNamara JR, Jenner JL, Ordovas JM, Wilson PWF and Schaefer EJ: Low density lipoprotein particle size and coronary artery disease. Arterioscler Thromb. 1992; 12: 187-195

23) McNamara JR, Jenner JL, Li Z, Wilson PW, and Schaefer EJ: Change in LDL particle size is associated with change in plasma triglyceride concentration. Arterioscler Thromb. 1992; 12: 1284-1290

24) Hirano T, Ito Y, Saegusa H, Yoshino G: A novel and simple method for quantification of small, dense LDL. J Lipid Res. 2003; 44: 2193-2201

25) Ito Y, Fujimura M, Ohta M, Hirano T: Development of a homogeneous assay for measurement of small dense LDL cholesterol. CLIN CHEM. 2011; 57: 57-65

26) Koba S, Yokota Y, Hirano T, Ito Y, Ban Y, Tsunoda F, Sato T, Shoji M, Suzuki H, Geshi E, Kobayashi Y and Katagiri T: Small LDL-cholesterol is superior to LDL-cholesterol for determining severe coronary atherosclerosis. J Atheroscler Thromb. 2008; 15: 250-260

27) Ai M, Otokozawa S, Asztalos BF, Ito Y, Nakajima K, White CC, Cupples LA, Wilson PW, and Schaefer EJ: Small dense LDL cholesterol and coronary heart disease: results from the Framingham Offspring Study. Clin Chem. 2010; 56: $967-976$

28) Arai H, Kokubo Y, Watanabe M, Sawamura T, Ito Y, Minagawa A, Okamura T, and Miyamato Y: Small dense low-density lipoproteins cholesterol can predict incident cardiovascular disease in an urban Japanese cohort: the Suita study. J Atheroscler Thromb. 2013; 20: 195-203

29) Hoogeveen RC, Gaubatz JW, Sun W, Dodge RC, Crosby JR, Jiang J, Couper D, Virani SS, Kathiresan S, Boerwinkle E, and Ballantyne CM: Small dense low-density lipoprotein-cholesterol concentrations predict risk for coronary heart disease: the Atherosclerosis Risk In Communities (ARIC) study. Arterioscler Thromb Vasc Biol. 2014; 34: 1069-1077

30) Tsai MY, Steffen BT, Guan W, McClelland RL, Warnick R, McConnell J, Hoefner DM, and Remaley AT: New automated assay of small dense low-density lipoprotein cholesterol identifies risk of coronary heart disease: the Multi-ethnic Study of Atherosclerosis. Arterioscler Thromb Vasc Biol. 2014; 34: 196-201

31) März W, Scharnagl H, Winkler K, Tiran A, Nauck M, Boehm BO, and Winkelmann BR: Low-density lipoprotein triglycerides associated with low-grade systemic inflam- 
mation, adhesion molecules, and angiographic coronary artery disease, the Ludwigshafen Risk and Cardiovascular Health study. Circulation. 2004; 110: 3068-3074

32) Ito Y, Ohta M, Ikezaki H, Hirao Y, Machida A, Schaefer EJ, and Furusyo N: Development and population results of a fully automated homogeneous assay for LDL triglyceride. JALM. 2017; 2: 746-756

33) Saeed A, Feofanova EV, Yu B, Sun W, Virani SS, Nambi V, Coresh J, Guild CS, Boerwinkle E, Ballantyne CM, and Hoogeveen RC: Remnant-Like Particle Cholesterol, Low-Density Lipoprotein Triglycerides, and Incident Cardiovascular Disease. J Am Coll Cardiol. 2018; 72: 156169

34) Hsu P, Ai M, Kanda E, Yu NC, Chen HL, Chen HW, Cheng MH, Kohzuma T, Schaefer EJ, and Yoshida M: A comparison of glycated albumin and glycosylated hemoglobin for the screening of diabetes mellitus in Taiwan. Atherosclerosis, 2015; 242: 327-333

35) Obesity definition: Health Promotion Administration. Ministry of Health and Welfare, Taiwan ROC. (http:// health99.hpa.gov.tw/OnlinkHealth/BMI.html) last access 3rd January 2019

36) Chang HC, Yang HC, Chang HY, Yeh CJ, Chen HH, Huang KC, and Pan WH. Morbid obesity in Taiwan: Prevalence, trends, associated social demographics, and lifestyle factors. PLoS ONE 2017; 12: e0169577

37) Japan Society for the Study of Obesity. New Criteria for 'Obesity Disease' in Japan. The Examination Committee of Criteria for 'Obesity Disease' in Japan. Circ J. 2002; 66: $987-992$

38) Otokozawa S, Ai M, Asztalos BF, White CC, DemissieBanjaw S, Cupples LA, Nakajima K, Wilson PWF, and Schaefer, E. J. Direct assessment of plasma low density lipoprotein and high density lipoprotein cholesterol levels and coronary heart disease: results from the Framingham Offspring Study. Atherosclerosis. 2010; 213: 251-255

39) Friedewald WT, Levy RI, Fredrickson DS. Estimation of the concentration of low-density lipoprotein cholesterol in plasma, without use of the preparative ultracentrifuge. Clin Chem. 1972; 18: 499-502

40) Hirao Y, Nakajima K, Machida T, Murakami M, Ito Y: Development of a novel homogeneous assay for remnant lipoprotein particle holesterol. JALM. 2018; 3: 26-36

41) Qi Y, Liu J, Wang W, Wang M, Zhao F, Sun J, Liu J and Zhao D: Apolipoprotein E-containing high-density lipo- protein (HDL) modifies the impact of cholesterol-overloaded HDL on incident coronary heart disease risk: A community-based cohort study. J Clin Lipidol. 2018; 12: 89-98

42) Ito Y, Satoh N, Ishii T, Kumakura J, and Hirano T: Development of a homogeneous assay for measurement of high-density lipoprotein-subclass cholesterol. Clin Chim Acta. 2014; 427: 86-93

43) Takahashi Y, Ito Y, Sakurai T, Wada N, Nagasaka A, Fujikawa M, Chiba H, and Hui SP: A Two-step homogeneous assay for apoE-containing HDL-cholesterol. Ann Clin Biochem. 2018: 56; 123-132

44) Turner RC, Holman RR, Matthews D, Hockaday TDR, and Peto J: Insulin deficiency and insulin resistance interaction in diabetes: estimation of their relative contribution by feedback analysis from basal plasma insulin and glucose concentrations. Metabolism. 1979; 28: 1086-1096

45) Matthews DR, Hosker JP, Rudenski AS, Naylor BA Treacher DF, and Turner RC: Homeostasis model assessment: insulin resistance and $\beta$-cell function from fasting plasma glucose and insulin concentrations in man. Diabetologia. 1985; 28: 412-419

46) Ai M, Otokozawa S, Asztalos BF, Nakajima K, Stein E, Jones PH, and Schaefer EJ: Effects of maximal doses of atorvastatin versus rosuvastatin on small dense low-density lipoprotein cholesterol levels. J Am Coll Cardiol. 2008; 101: 315-318

47) Ikezaki H, Ai M, Schaefer EJ, Otokozawa S, Asztalos BF, Nakajima K, Zhou Y, Liu CT, Jacques PF, Cupples LA, and Furusyo N: Ethnic differences in glucose homeostasis markers between the Kyushu-Okinawa Population Study and the Framingham Offspring Study. Sci Rep. 2016; 6: 36725

48) Ikezaki H, Ai M, Schaefer EJ, Otokozawa S, Asztalos BF, Nakajima K, Zhou Y, Liu CT, Jacques PF, Cupples LA, and Furusyo N: Cardiovascular disease prevalence and insulin resistance in the Kyushu-Okinawa Population Study and the Framingham Offspring Study. J Clin Lipidol. 2017; 11: 348-356

49) Furusyo N, Ai M, Okazaki M, Ikezaki H, Ihara T, Hayashi T, Hiramine S, Ura K, Kohzuma T, Schaefer EJ and Hayashi J: Serum cholesterol and triglyceride reference ranges of twenty lipoprotein subclasses for healthy Japanese men and women. Atherosclerosis, 2013; 231: 238245 


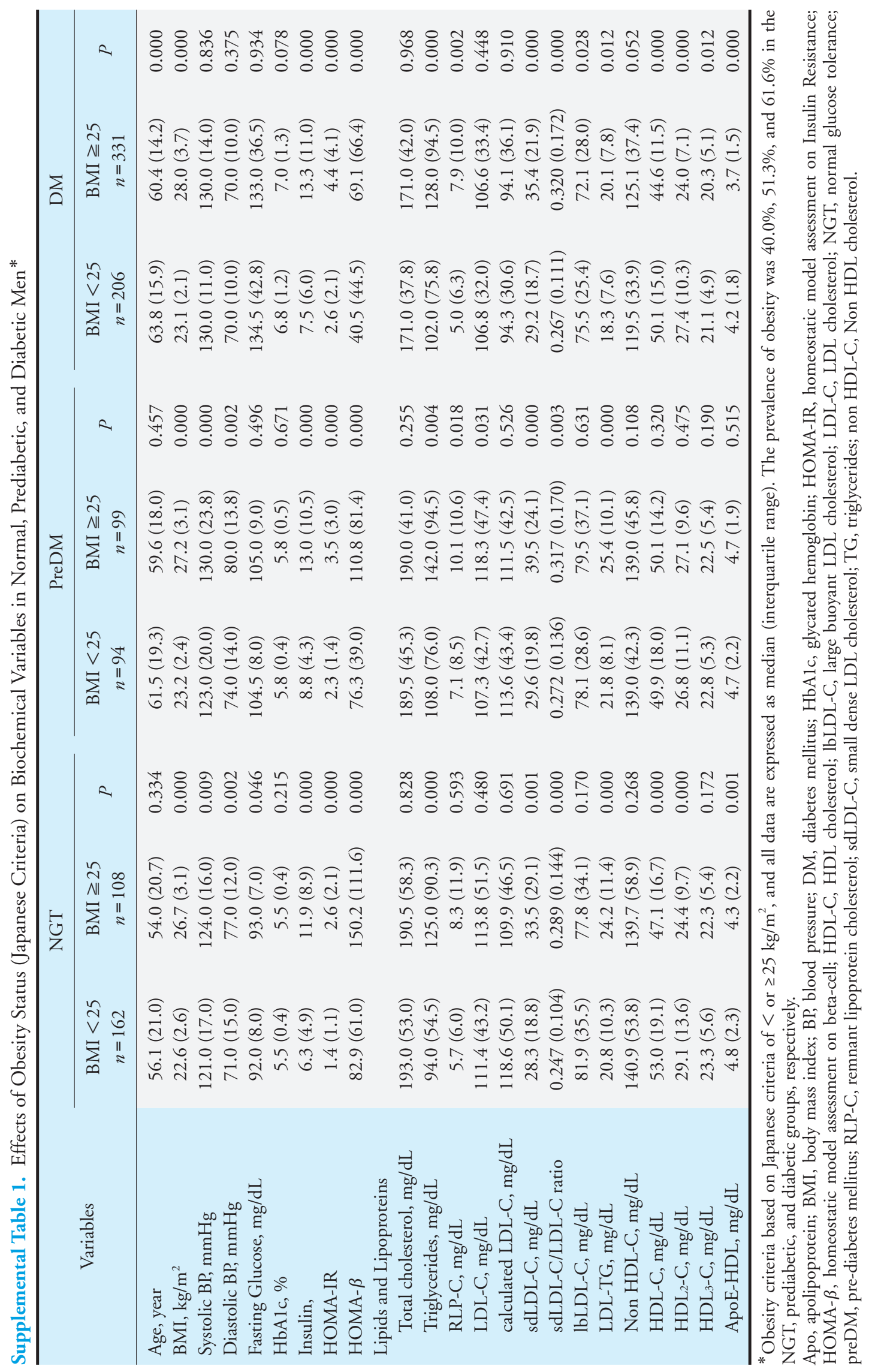




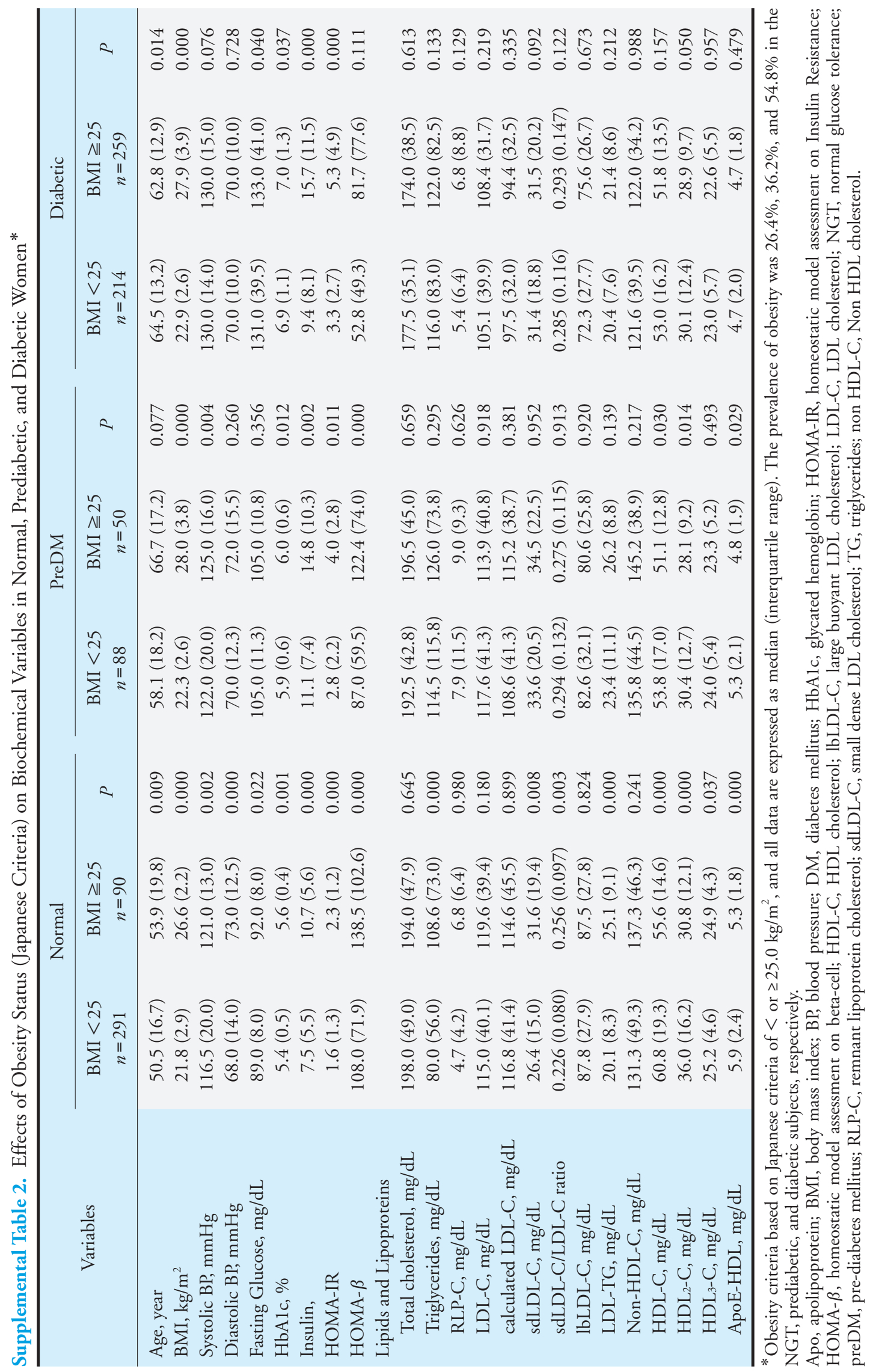


Supplemental Table 3. Comparison of variables between normal participants with or without using statin*

\begin{tabular}{|c|c|c|c|c|c|c|}
\hline \multirow[b]{2}{*}{ Variables } & \multicolumn{2}{|c|}{ Male Participants } & \multirow[b]{2}{*}{$p$} & \multicolumn{2}{|c|}{ Female Participants } & \multirow[b]{2}{*}{$p$} \\
\hline & $\begin{array}{l}\text { Statin - } \\
(n=207)\end{array}$ & $\begin{array}{l}\text { Statin }+ \\
(n=62)\end{array}$ & & $\begin{array}{l}\text { Statin - } \\
(n=324)\end{array}$ & $\begin{array}{l}\text { Statin }+ \\
(n=60)\end{array}$ & \\
\hline Age, year & $52.2(18.3)$ & $66.0(16.2)$ & 0.000 & $49.3(12.9)$ & $69.5(13.0)$ & 0.000 \\
\hline BMI, $\mathrm{kg} / \mathrm{m}^{2}$ & $23.9(4.1)$ & $24.7(3.4)$ & 0.044 & $22.5(4.0)$ & $24.0(4.1)$ & 0.001 \\
\hline Obesity Prevalence & $36(17.4 \%)^{* *}$ & $14(22.6 \%)^{* *}$ & 0.009 & $25(7.7 \%)^{* *}$ & $10(16.7 \%)^{* *}$ & 0.003 \\
\hline Systolic BP, mmHg & $122.0(15.8)$ & $130.0(15.3)$ & 0.097 & $117.0(19.0)$ & $129.5(18.3)$ & 0.000 \\
\hline Diastolic BP, mmHg & $74.0(13.0)$ & $75.0(10.8)$ & 0.348 & $68.0(14.0)$ & $76.0(10.5)$ & 0.020 \\
\hline Fasting Glucose, $\mathrm{mg} / \mathrm{dL}$ & $92.0(7.5)$ & $94.0(7.0)$ & 0.182 & $89.5(8.0)$ & $92.0(8.5)$ & 0.000 \\
\hline HbA1c, \% & $5.5(0.4)$ & $5.6(0.3)$ & 0.584 & $5.4(0.4)$ & $5.6(0.5)$ & 0.000 \\
\hline Insulin, & $7.9(7.4)$ & $8.5(6.8)$ & 0.494 & $7.9(5.6)$ & $10.1(7.6)$ & 0.004 \\
\hline HOMA-IR & $1.8(1.6)$ & $1.9(1.5)$ & 0.578 & $1.8(1.3)$ & $2.3(1.7)$ & 0.001 \\
\hline НОМА- $\beta$ & $99.5(96.2)$ & $104.0(68.5)$ & 0.271 & $110.7(76.0)$ & $128.9(85.5)$ & 0.483 \\
\hline \multicolumn{7}{|l|}{ Lipids and Lipoproteins } \\
\hline Total cholesterol, $\mathrm{mg} / \mathrm{dL}$ & $196.1(50.5)$ & $175.5(57.2)$ & 0.056 & $198.0(47.3)$ & $193.8(56.0)$ & 0.767 \\
\hline Triglycerides, mg/dL & $103.0(79.5)$ & $100.5(47.3)$ & 0.260 & $81.0(57.5)$ & $107.6(68.3)$ & 0.000 \\
\hline RLP-C, mg/dL & $6.3(8.2)$ & $5.7(5.6)$ & 0.309 & $4.8(4.2)$ & $7.4(5.8)$ & 0.842 \\
\hline $\mathrm{LDL}-\mathrm{C}, \mathrm{mg} / \mathrm{dL}$ & $116.7(44.8)$ & $102.1(46.0)$ & 0.194 & $117.7(39.2)$ & $112.5(40.6)$ & 0.285 \\
\hline calculated LDL-C, mg/dL & $119.2(44.3)$ & $100.6(51.9)$ & 0.078 & $117.3(40.7)$ & $111.2(52.1)$ & 0.342 \\
\hline sdLDL-C, mg/dL & $30.7(22.9)$ & $27.5(15.0)$ & 0.216 & $26.5(16.8)$ & $30.0(17.2)$ & 0.218 \\
\hline sdLDL-C/LDL-C ratio & $0.256(0.118)$ & $0.265(0.070)$ & 0.460 & $0.226(0.081)$ & $0.278(0.091)$ & 0.000 \\
\hline lbLDL-C, mg/dL & $84.9(33.0)$ & $76.0(32.7)$ & 0.324 & $89.8(27.4)$ & $81.7(31.4)$ & 0.019 \\
\hline LDL-TG, mg/dL & $22.1(11.3)$ & $22.0(8.2)$ & 0.419 & $20.1(9.9)$ & $23.8(6.1)$ & 0.001 \\
\hline Non HDL-C, mg/dL & $142.9(52.2)$ & $122.9(51.7)$ & 0.062 & $133.4(48.1)$ & $135.9(53.9)$ & 0.696 \\
\hline HDL-C, mg/dL & $51.6(18.1)$ & $49.9(16.0)$ & 0.729 & $59.9(18.8)$ & $58.2(15.4)$ & 0.088 \\
\hline $\mathrm{HDL}_{2}-\mathrm{C}, \mathrm{mg} / \mathrm{dL}$ & $27.6(13.0)$ & $26.4(12.9)$ & 0.961 & $34.7(16.1)$ & $32.1(11.1)$ & 0.074 \\
\hline $\mathrm{HDL}_{3}-\mathrm{C}, \mathrm{mg} / \mathrm{dL}$ & $23.0(5.8)$ & $22.8(4.2)$ & 0.279 & $25.1(4.7)$ & 24.9 (3.9) & 0.534 \\
\hline ApoE-HDL, mg/dL & $4.6(2.1)$ & $4.5(2.5)$ & 0.962 & $5.7(2.3)$ & $5.8(2.0)$ & 0.354 \\
\hline
\end{tabular}

* Data are expressed as median (interquartile range).

** Data are expressed as number of obese participant (percentage of obese participants) (BMI $\geq 27 \mathrm{~kg} / \mathrm{m}^{2}$ ).

Apo, apolipoprotein; BMI, body mass index; BP, blood pressure; DM, diabetes mellitus; HbA1c, glycated hemoglobin; HOMA-IR, homeostatic model assessment on Insulin Resistance; HOMA- $\beta$, homeostatic model assessment on beta-cell; HDL-C, HDL cholesterol; lbLDL-C, large buoyant LDL cholesterol; LDL-C, LDL cholesterol; NGT, normal glucose tolerance; preDM, pre-diabetes mellitus; RLP-C, remnant lipoprotein cholesterol; sdLDL-C, small dense LDL cholesterol; TG, triglycerides 
Supplemental Table 4. Comparison of variables between prediabetic participants with or without using statin*

\begin{tabular}{|c|c|c|c|c|c|c|}
\hline \multirow[b]{2}{*}{ Variables } & \multicolumn{2}{|c|}{ Male Participants } & \multirow[b]{2}{*}{$p$} & \multicolumn{2}{|c|}{ Female Participants } & \multirow[b]{2}{*}{$p$} \\
\hline & $\begin{array}{l}\text { Statin - } \\
(n=117)\end{array}$ & $\begin{array}{l}\text { Statin }+ \\
(n=79)\end{array}$ & & $\begin{array}{l}\text { Statin - } \\
(n=90)\end{array}$ & $\begin{array}{l}\text { Statin + } \\
(n=52)\end{array}$ & \\
\hline Age, year & $54.5(18.5)$ & $66.0(12.7)$ & 0.000 & $54.7(19.2)$ & $68.0(12.8)$ & 0.000 \\
\hline BMI, $\mathrm{kg} / \mathrm{m}^{2}$ & $25.3(4.0)$ & $25.0(4.1)$ & 0.067 & $24.1(5.2)$ & $23.5(4.5)$ & 0.079 \\
\hline Obesity Prevalence & $36(30.8 \%)^{* *}$ & $16(20.3 \%)^{* *}$ & 0.888 & $23(25.5 \%)^{* *}$ & $8(15.4 \%)^{* *}$ & 0.371 \\
\hline Systolic BP, mmHg & $130.0(22.0)$ & $127.0(12.0)$ & 0.309 & $120.0(17.0)$ & $130.0(10.0)$ & 0.041 \\
\hline Diastolic BP, mmHg & $77.0(17.0)$ & $75.0(11.0)$ & 0.170 & $70.5(11.8)$ & $77.0(12.0)$ & 0.136 \\
\hline Fasting Glucose, mg/dL & $105.0(10.0)$ & $106.0(8.5)$ & 0.563 & $105.0(11.0)$ & $104.5(10.3)$ & 0.158 \\
\hline HbA1c, \% & $5.8(0.5)$ & $5.9(0.4)$ & 0.807 & $5.8(0.7)$ & $5.9(0.4)$ & 0.544 \\
\hline Insulin, & $10.6(8.9)$ & $11.2(8.0)$ & 0.319 & $11.9(8.2)$ & $13.4(10.2)$ & 0.566 \\
\hline HOMA-IR & $2.7(2.3)$ & $3.0(2.1)$ & 0.449 & $3.2(2.2)$ & $3.4(2.6)$ & 0.899 \\
\hline НOMA- $\beta$ & $85.1(63.5)$ & $90.4(51.9)$ & 0.254 & $95.7(64.5)$ & $116.4(75.1)$ & 0.065 \\
\hline \multicolumn{7}{|l|}{ Lipids and Lipoproteins } \\
\hline Total cholesterol, mg/dL & $192.0(32.0)$ & $184.0(56.0)$ & 0.306 & $194.5(38.5)$ & $193.5(54.5)$ & 0.234 \\
\hline Triglycerides, mg/dL & $133.0(99.0)$ & $111.0(69.0)$ & 0.119 & $106.5(82.8)$ & $131.5(106.3)$ & 0.002 \\
\hline RLP-C, mg/dL & $10.1(11.6)$ & $7.4(8.3)$ & 0.029 & $7.0(8.4)$ & $9.5(14.6)$ & 0.012 \\
\hline LDL-C, mg/dL & $115.5(40.9)$ & $106.7(48.8)$ & 0.579 & $118.1(39.5)$ & $110.0(49.0)$ & 0.124 \\
\hline calculated LDL-C, mg/dL & $114.1(38.0)$ & $106.9(47.7)$ & 0.912 & $113.5(36.2)$ & $106.3(55.2)$ & 0.055 \\
\hline sdLDL-C, mg/dL & $34.9(26.1)$ & $33.4(22.4)$ & 0.369 & $32.5(22.5)$ & $35.8(21.4)$ & 0.271 \\
\hline sdLDL-C/LDL-C ratio & $0.310(0.164)$ & $0.288(0.121)$ & 0.356 & $0.272(0.103)$ & $0.330(0.136)$ & 0.002 \\
\hline $\mathrm{lbLDL}-\mathrm{C}, \mathrm{mg} / \mathrm{dL}$ & $79.7(32.5)$ & $73.8(31.0)$ & 0.902 & $84.4(22.7)$ & $72.2(29.5)$ & 0.004 \\
\hline LDL-TG, mg/dL & $23.4(10.9)$ & $22.9(8.8)$ & 0.259 & $23.3(10.6)$ & $26.9(10.1)$ & 0.168 \\
\hline Non HDL-C, mg/dL & $141.6(33.2)$ & $131.1(50.3)$ & 0.320 & $139.7(38.8)$ & $138.5(54.9)$ & 0.614 \\
\hline HDL-C, mg/dL & $51.4(18.0)$ & $49.4(13.8)$ & 0.846 & $53.6(16.7)$ & $51.0(12.6)$ & 0.054 \\
\hline $\mathrm{HDL}_{2}-\mathrm{C}, \mathrm{mg} / \mathrm{dL}$ & $26.3(12.4)$ & $27.1(7.9)$ & 0.851 & $29.8(13.7)$ & $26.1(9.1)$ & 0.059 \\
\hline $\mathrm{HDL}_{3}-\mathrm{C}, \mathrm{mg} / \mathrm{dL}$ & $22.9(5.5)$ & $22.5(5.3)$ & 0.888 & $23.9(5.4)$ & $22.8(4.9)$ & 0.205 \\
\hline ApoE-HDL, mg/dL & $4.8(2.1)$ & $4.6(1.9)$ & 0.919 & $5.2(2.3)$ & $4.9(1.4)$ & 0.259 \\
\hline
\end{tabular}

* Data are expressed as median (interquartile range).

** Data are expressed as number of obese participant (percentage of obese participants) (BMI $\geq 27 \mathrm{~kg} / \mathrm{m}^{2}$ ).

Apo, apolipoprotein; BMI, body mass index; BP, blood pressure; DM, diabetes mellitus; HbA1c, glycated hemoglobin; HOMA-IR, homeostatic model assessment on Insulin Resistance; HOMA- $\beta$, homeostatic model assessment on beta-cell; HDL-C, HDL cholesterol; lbLDL-C, large buoyant LDL cholesterol; LDL-C, LDL cholesterol; NGT, normal glucose tolerance; preDM, pre-diabetes mellitus; RLP-C, remnant lipoprotein cholesterol; sdLDL-C, small dense LDL cholesterol; TG, triglycerides 
Supplemental Table 5. Comparison of variables between diabetic participants with or without using statin*

\begin{tabular}{|c|c|c|c|c|c|c|}
\hline \multirow[b]{2}{*}{ Variables } & \multicolumn{2}{|c|}{ Male Participants } & \multirow[b]{2}{*}{$p$} & \multicolumn{2}{|c|}{ Female Participants } & \multirow[b]{2}{*}{$p$} \\
\hline & $\begin{array}{l}\text { Statin - } \\
(n=281)\end{array}$ & $\begin{array}{l}\text { Statin }+ \\
(n=206)\end{array}$ & & $\begin{array}{l}\text { Statin - } \\
(n=208)\end{array}$ & $\begin{array}{l}\text { Statin }+ \\
(n=227)\end{array}$ & \\
\hline Age, year & $61.9(13.4)$ & $62.4(15.2)$ & 0.521 & $63.6(11.9)$ & $64.3(13.1)$ & 0.286 \\
\hline BMI, $\mathrm{kg} / \mathrm{m}^{2}$ & $25.4(4.7)$ & $26.6(4.9)$ & 0.007 & $24.8(4.8)$ & $26.2(5.4)$ & 0.001 \\
\hline Obesity Prevalence & $94(33.5 \%)^{* *}$ & $88(42.7 \%)^{* *}$ & 0.001 & $57(27.4 \%)^{* *}$ & $94(41.4 \%)^{* *}$ & 0.058 \\
\hline Systolic BP, mmHg & $130.0(14.0)$ & $130.0(10.0)$ & 0.945 & $130.0(19.5)$ & $130.0(10.0)$ & 0.352 \\
\hline Diastolic BP, mmHg & $76.0(10.0)$ & $70.0(10.0)$ & 0.419 & $70.0(10.0)$ & $70.0(10.0)$ & 0.257 \\
\hline Fasting Glucose, mg/dL & $134.0(35.0)$ & $132.0(39.8)$ & 0.311 & $132.0(39.5)$ & $131.0(42.0)$ & 0.618 \\
\hline HbA1c, \% & $6.9(1.1)$ & $6.8(1.4)$ & 0.657 & $7.0(1.2)$ & $6.9(1.3)$ & 0.189 \\
\hline Insulin, & $10.5(10.7)$ & $10.4(7.9)$ & 0.495 & $12.0(9.4)$ & $13.7(10.5)$ & 0.644 \\
\hline HOMA-IR & $3.3(3.7)$ & $3.5(3.0)$ & 0.611 & $4.0(3.6)$ & $4.6(3.9)$ & 0.757 \\
\hline НОМА- $\beta$ & $55.2(61.7)$ & $60.0(56.7)$ & 0.522 & $60.9(60.0)$ & $69.2(72.7)$ & 0.155 \\
\hline \multicolumn{7}{|l|}{ Lipids and Lipoproteins } \\
\hline Total cholesterol, mg/dL & $171.0(33.0)$ & $168.0(50.8)$ & 0.214 & $178.0(31.1)$ & $172.0(41.0)$ & 0.971 \\
\hline Triglycerides, mg/dL & $116.0(91.0)$ & $118.0(88.3)$ & 0.579 & $118.0(75.0)$ & $120.0(78.0)$ & 0.126 \\
\hline RLP-C, mg/dL & $5.6(8.0)$ & $7.3(9.4)$ & 0.133 & $5.3(6.1)$ & $6.8(8.7)$ & 0.073 \\
\hline $\mathrm{LDL}-\mathrm{C}, \mathrm{mg} / \mathrm{dL}$ & $107.2(30.1)$ & $104.7(40.9)$ & 0.087 & $108.4(29.7)$ & $104.1(39.6)$ & 0.655 \\
\hline calculated LDL-C, mg/dL & $94.1(30.9)$ & $92.7(40.5)$ & 0.141 & $101.0(27.1)$ & $91.3(34.7)$ & 0.099 \\
\hline sdLDL-C, mg/dL & $31.8(18.8)$ & $33.2(21.0)$ & 0.056 & $29.4(19.9)$ & $32.8(16.9)$ & 0.039 \\
\hline sdLDL-C/LDL-C ratio & $0.288(0.139)$ & $0.304(0.140)$ & 0.298 & $0.272(0.124)$ & $0.301(0.131)$ & 0.011 \\
\hline lbLDL-C, mg/dL & $74.6(25.2)$ & $72.4(26.8)$ & 0.394 & $76.4(24.1)$ & $71.7(27.8)$ & 0.304 \\
\hline LDL-TG, mg/dL & $18.1(6.6)$ & $20.0(8.1)$ & 0.003 & $19.3(7.9)$ & $21.7(8.6)$ & 0.000 \\
\hline Non HDL-C, mg/dL & $122.8(30.9)$ & $119.5(44.6)$ & 0.257 & $124.4(33.5)$ & $117.3(36.8)$ & 0.454 \\
\hline HDL-C, mg/dL & $46.0(13.6)$ & $46.8(14.1)$ & 0.587 & $50.3(17.2)$ & $54.0(13.9)$ & 0.034 \\
\hline $\mathrm{HDL}_{2}-\mathrm{C}, \mathrm{mg} / \mathrm{dL}$ & $25.2(8.3)$ & $25.4(8.4)$ & 0.736 & $28.4(11.8)$ & $30.3(10.2)$ & 0.144 \\
\hline $\mathrm{HDL}_{3}-\mathrm{C}, \mathrm{mg} / \mathrm{dL}$ & $20.5(5.1)$ & $20.9(5.3)$ & 0.409 & $22.2(5.2)$ & $23.4(5.3)$ & 0.002 \\
\hline ApoE-HDL, mg/dL & $3.7(1.5)$ & $4.1(1.8)$ & 0.039 & $4.4(2.0)$ & $5.0(1.8)$ & 0.000 \\
\hline
\end{tabular}

* Data are expressed as median (interquartile range).

** Data are expressed as number of obese participant (percentage of obese participants) (BMI $\geq 27 \mathrm{~kg} / \mathrm{m}^{2}$ ).

Apo, apolipoprotein; BMI, body mass index; BP, blood pressure; DM, diabetes mellitus; HbA1c, glycated hemoglobin; HOMA-IR, homeostatic model assessment on Insulin Resistance; HOMA- $\beta$, homeostatic model assessment on beta-cell; HDL-C, HDL cholesterol; lbLDL-C, large buoyant LDL cholesterol; LDL-C, LDL cholesterol; NGT, normal glucose tolerance; preDM, pre-diabetes mellitus; RLP-C, remnant lipoprotein cholesterol; sdLDL-C, small dense LDL cholesterol; TG, triglycerides 


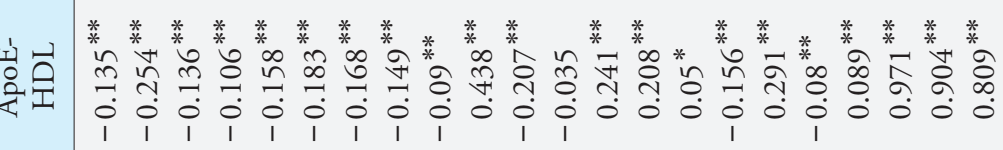

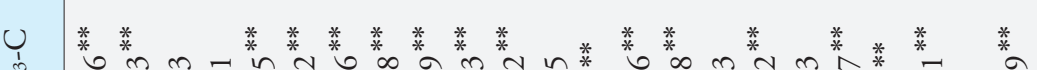

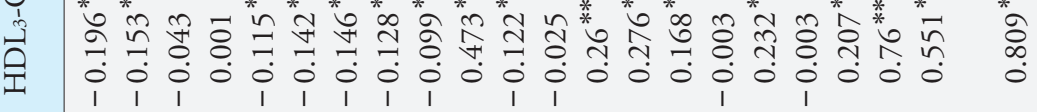

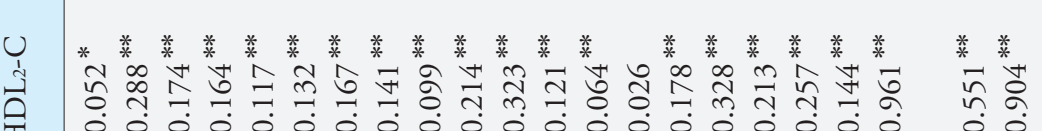

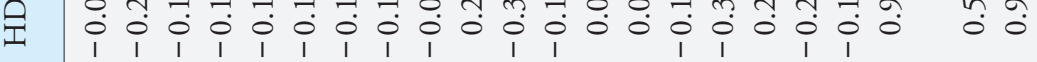

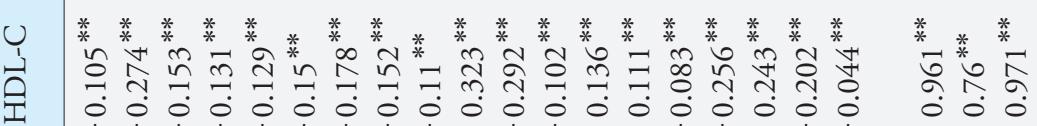

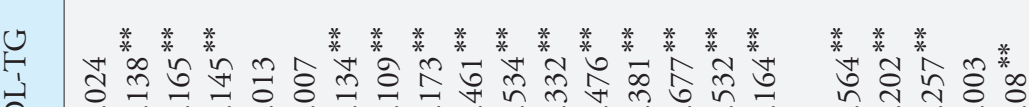

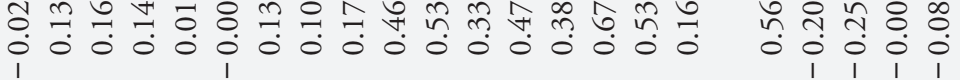

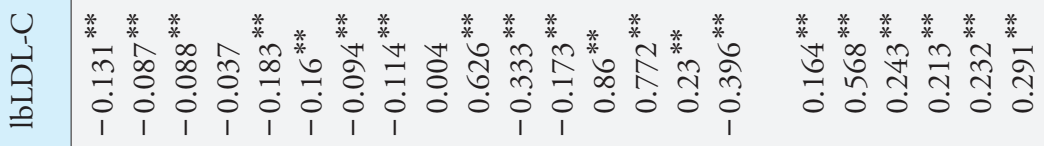

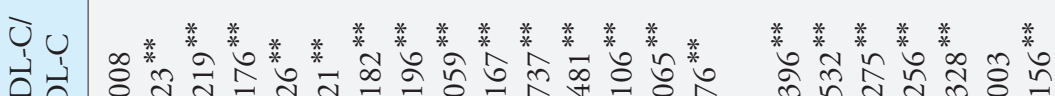

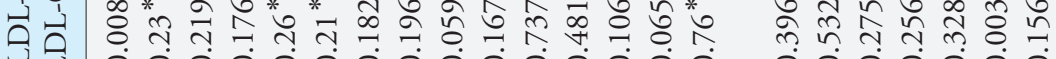

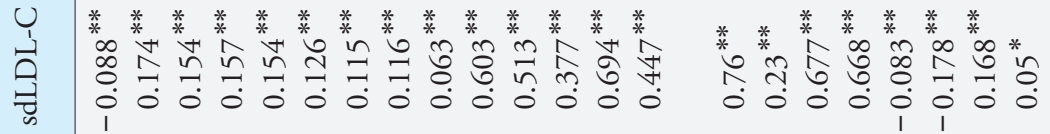

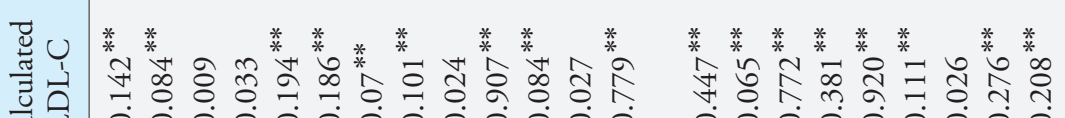

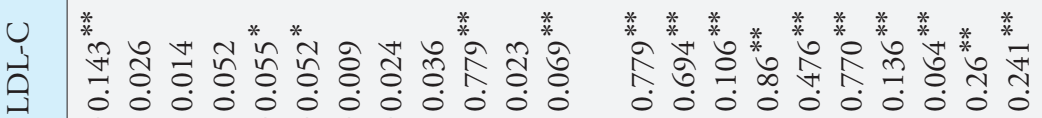

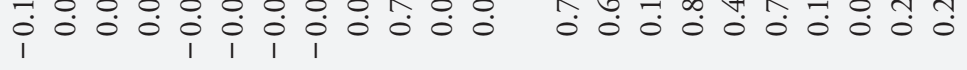

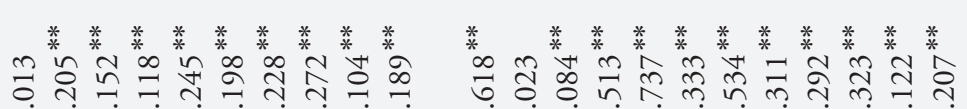

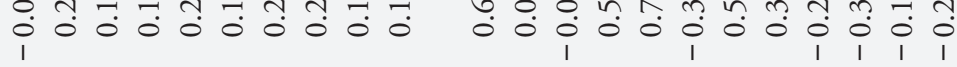

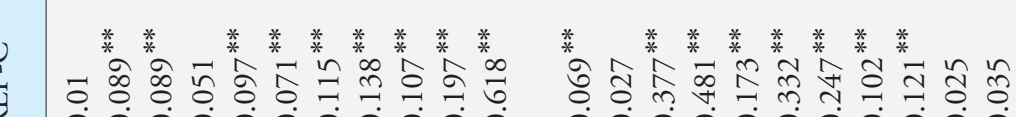

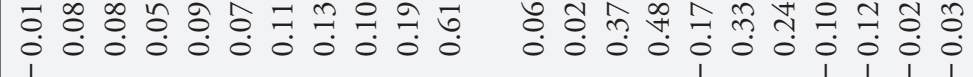

êt

至范

نิ

苟

$\exists$

芩

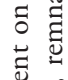

要

चั

.

苟

论

兟

范范

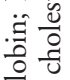

임

㘂

क्षे

苮

王

氙完

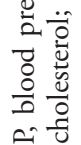

की

突宣

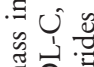

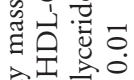

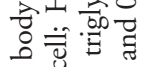

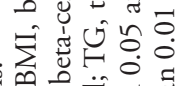

m.

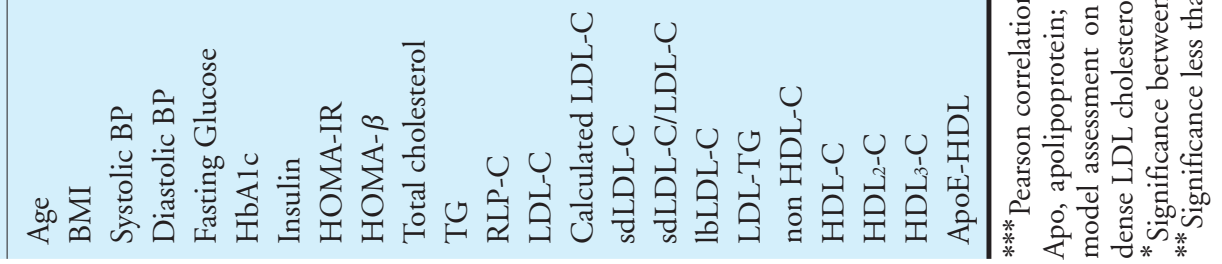




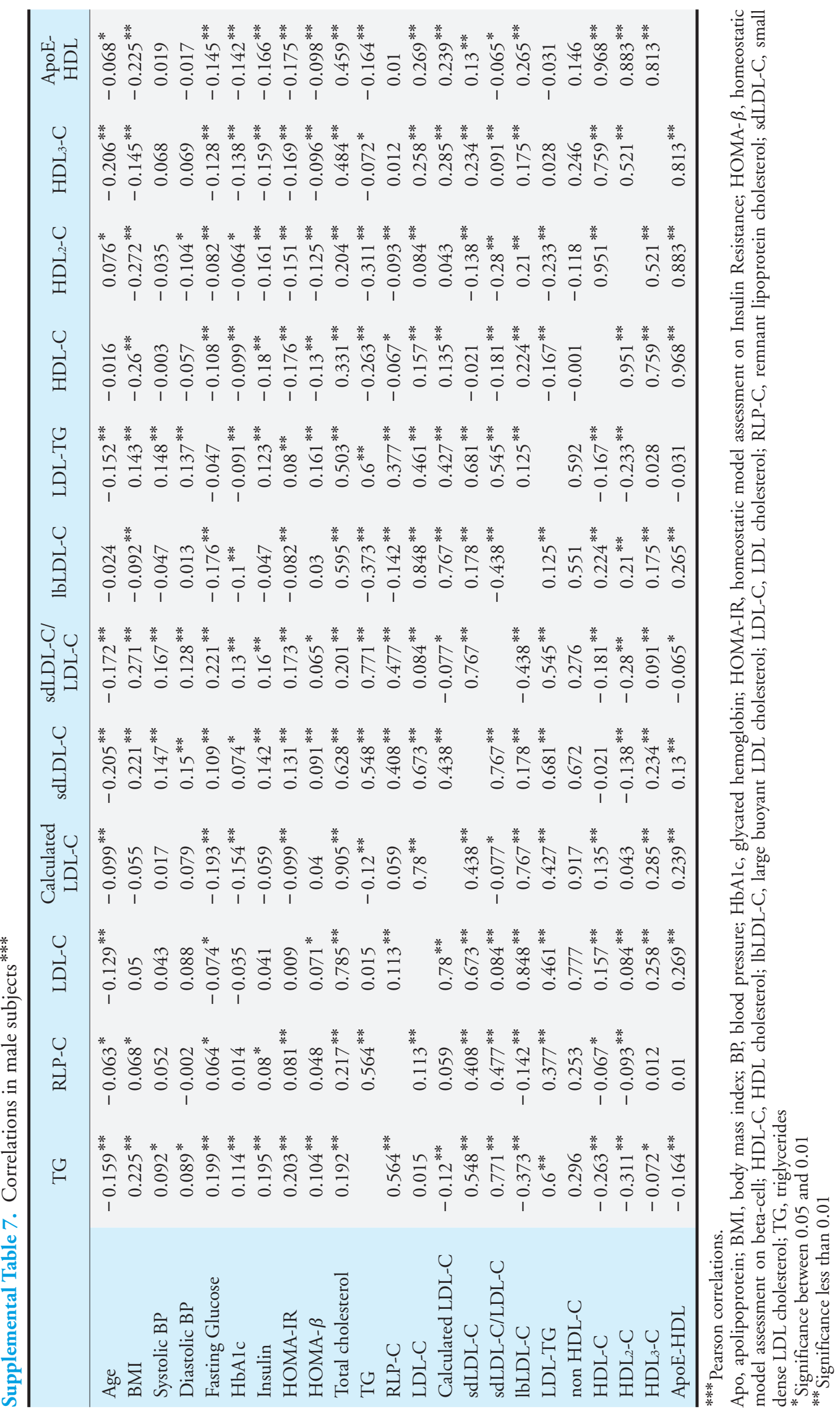




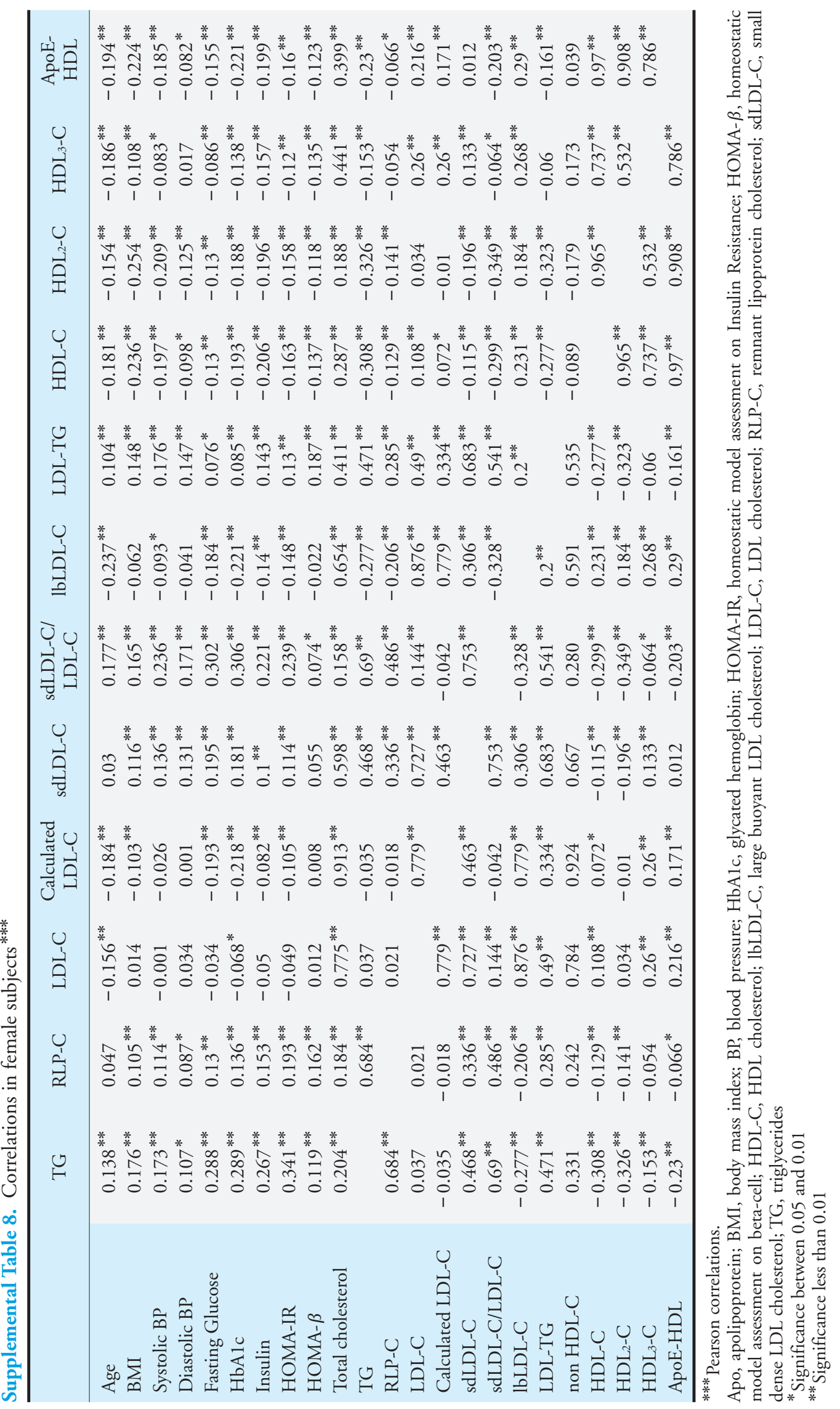

